UCRL-ID-123426

\title{
POPOVER Review Panel Report
}
A. Davito
C. J. Baker
C. J. King
B. Costerus
T. Nelson
D. Prokosch
J. Pastrnak
P. Grace

April 10, 1996

This is an informal report intended primarily for internal or limited external distribution. The opinions and conclusions stated are those of the author and may or may not be those of the Laboratory.

Work performed under the auspices of the U.S. Department of Energy by the

A Lawrence Livermore National Laboratory under Contract W-7405-Eng-48.

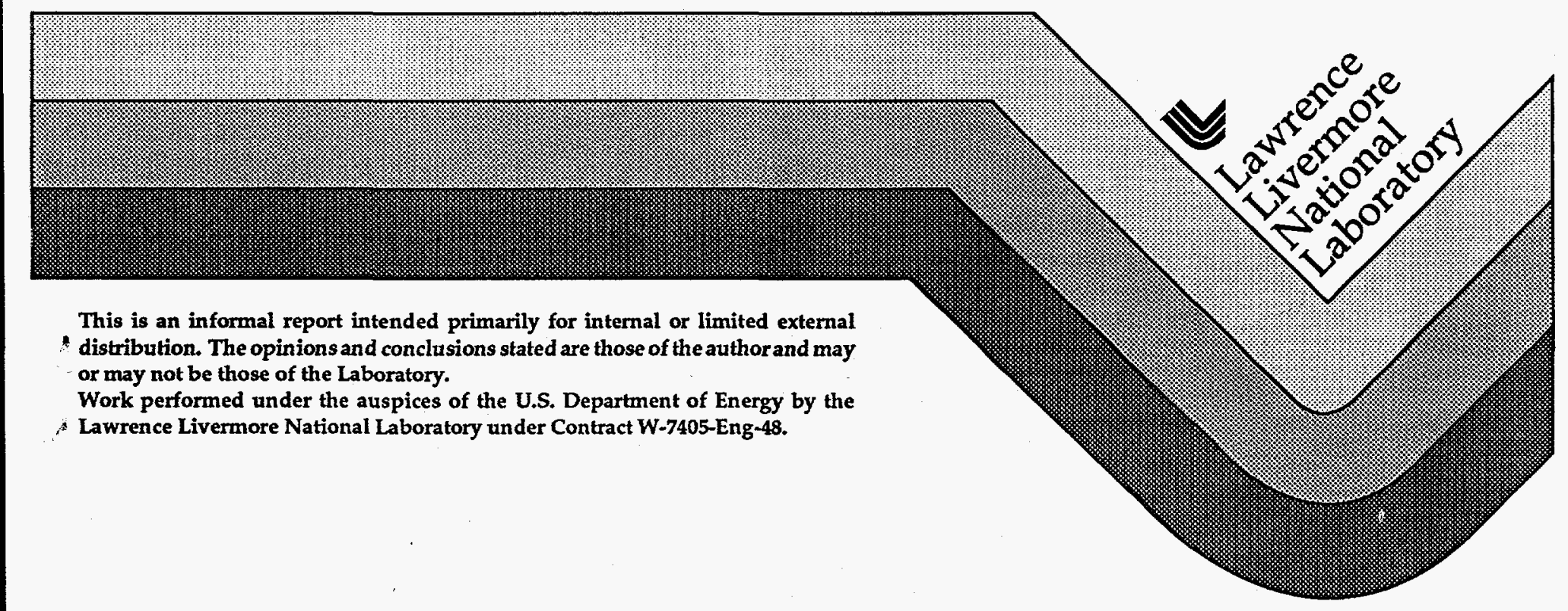




\section{DISCLAIMER}

Portions of this document may be illegible in electronic image products. Images are produced from the best available original document. 
Interdepartmental letterhead

Mall Station L- 113

Ext: $3-3583$

April 10, 1996

NTED96-21

TO: G. Mara / W. Cooper

FROM :

A. Davito

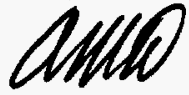

SUBJECT: POPOVER Panel Report

Attached is the completed POPOVER Review Panel Report. This report and two companion documents describe the POPOVER test series conducted at the Big Explosives Experiment Facility (BEEF). The companion documents are:

1) EG\&G Energy Measurements, POPOVER Certification Test Series Final Engineering Report, EG\&G Energy Measurements (EG\&GEM), Las Vegas, NV, SM:95E-179-38(1995). This three volume report documents the variety of engineering measurements made by EG\&GEM. These three volumes are in the Reports Library in Building 121 (ext. 25289).

2) R. Heinle and T. Stubbs, POPOVER Data Report, Lawrence Livermore National Laboratory, Livermore, CA, UCRL-ID123701, March 27, 1996. This report is a collection of the final data plots.

Attachment

Copies to:

C. "Joe" Baker, L-871

W. Bellow, BN e NLV 065

R. Berlo, L-667

G. Carlson, L-113

G. Christensen, L-777, 2 copies

B. Costerus, L-125

L. Davies, BN e NLV 065

B. Goodwin, L-35

Chi "John" King, L-654

P. Grace, L-871

R. Jandrisevits, L-125

L. Loquist, L-154

T. Nelson, $L-126$

J. Pastrnak, L-122

K. Pedersen, L-142

D. Prokosch, L-281

R. Robinson, BN \& NLV 065

C. Tinoco, L-658, 11 copies

$\mathrm{J}$. Wobser, $\mathrm{L}-170$

University of California

1 Lawrence Livermore National Laboratory

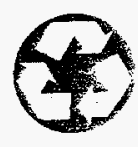


UCRL-ID-123426

\title{
POPOVER Review Panel Report
}

\author{
March 1996
}

\section{Approved:}

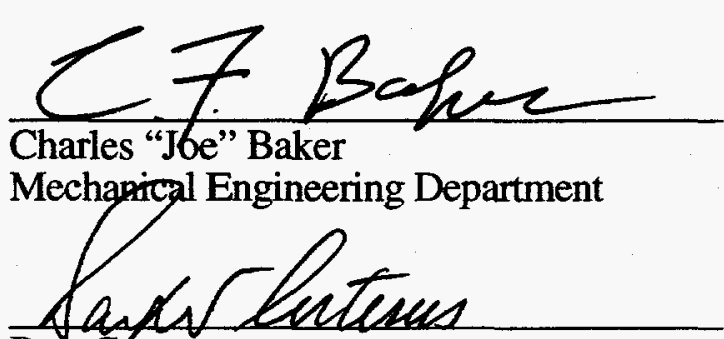

Bart Costerus

Defense Technologies Engineering Division

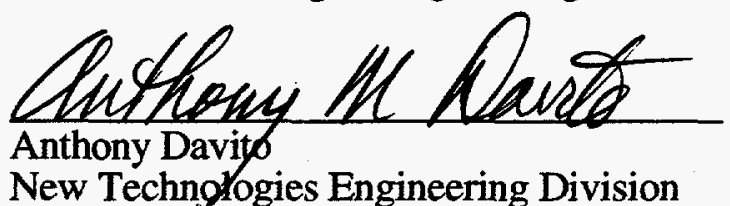

New Technologies Engineering Division

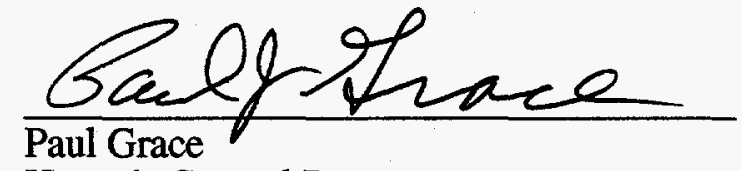

Hazards Control Department
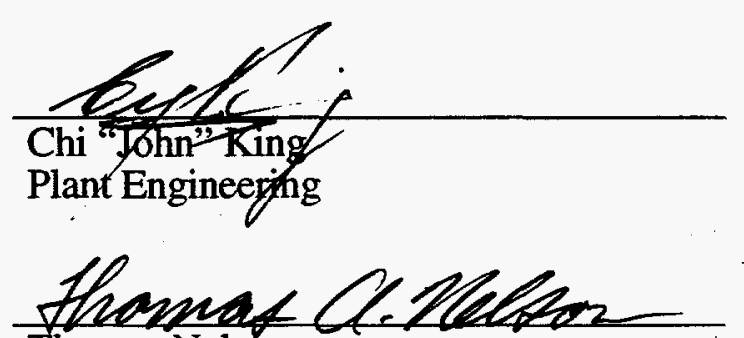
Thomas Nelson

New Technologies Engineering Division

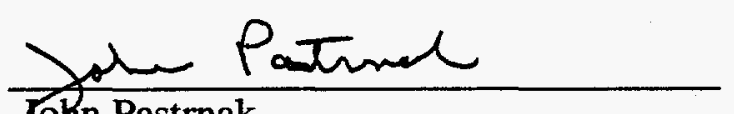

Zohn Pastrnak

Defense Technologies Engineering Division

Daid W. Prokosch David Prokosch

Hazards Control Department 


\section{POPOVER Review Panel Report}

\section{Contents}

Section 1. Executive Summary …..............................................................1-1

Section 2. The POPOVER Review Panel .................................................2-1

Charter of the Review Panel ...................................................................

Overview of the Review Panel Report..............................................................2-2

Section 3. Introduction to BEEF and POPOVER ........................................1

Background Information ................................................................................. 3-1

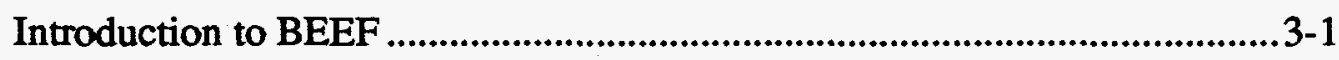

Introduction to POPOVER ........................................................................ 3-2

Section 4. Minimum Explosive Safety Requirements .....................................4-1

DOE Requirements ................................................................................

OSHA Requirement …...................................................................................... 4-2

Section 5. POPOVER Instrumentation and Data ........................................5-1

Section 6. Analysis of the POPOVER Test Series ........................................6-1

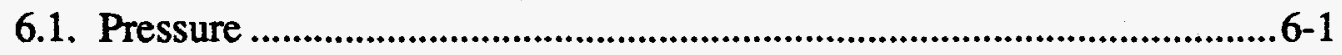

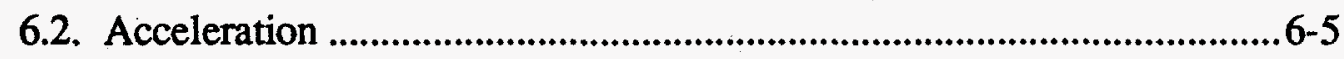

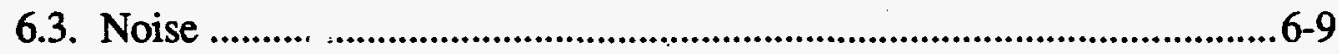

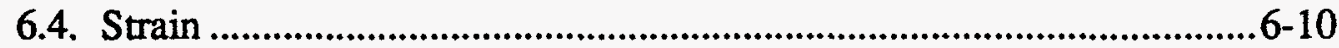

6.5. Blast Thermal Effects and Fragment Protection ....................................6-14

Section 7. Findings and Recommendations ................................................. 7-1

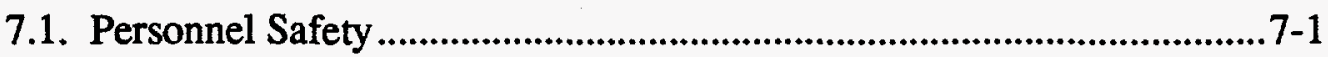

7.2. Facility and Equipment ........................................................................... 7-2

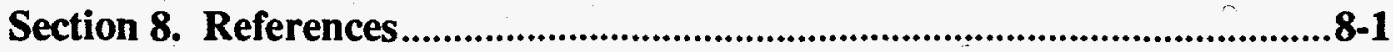




\section{Section 1}

\section{Executive Summary}

The POPOVER series of high explosive (HE) certification tests was conducted at the Big Explosives Experimental Facility (BEEF) in Area 4 of the Nevada Test Site (NTS). The two primary objectives of POPOVER were to certify that:

1. BEEF meets DOE requirements for explosives facilities and is safe for personneloccupied operations during testing of large charges of conventional HE.

2. Facility structures and equipment will function as intended when subjected to the effects of these charges.

After careful analysis of test results, the POPOVER Review Panel concludes that the POPOVER series met both objectives. Further details on the Review Panel's conclusions are included in Section 7 - Findings and Recommendations. 


\section{Section 2}

\section{The POPOVER Review Panel}

The purpose of the POPOVER test series was to measure and collect data from experiments designed to verify the integrity of the Big Explosive Experiment Facility (BEEF). The ultimate goal of these tests was to gain absolute confidence in the integrity of BEEF's structures and equipment and to allow personnel to operate in that facility's Bunker B4-300 during future experiments.

To attain this goal, an independent assessment of the POPOVER test series was carried out by assembling a group of mechanical, civil, and instrumentation engineers and experts in explosives safety - the POPOVER Review Panel. The Review Panel members and their areas of expertise were:

C. F. (Joe) Baker (civil and blast effects)

Bart W. Costerus (instrumentation)

Anthony M. Davito, chairman (structural)

Paul J. Grace (explosives safety)

Chi Yung (John) King (structural and blast effects)

Thomas A. Nelson (structural mechanics)

John W. Pastrnak (mechanical and blast effects)

David W. Prokosch (explosives safety)

This report documents the evaluation of BEEF by the POPOVER Review Panel.

\section{Charter of the Review Panel}

The Review Panel's evaluation process included the following tasks:

- Review the diagnostic plan for each POPOVER experiment to verify that it was a valid approach to providing confirming data.

- Review and evaluate the preliminary data resulting from each experiment in the POPOVER series and recommend any desirable changes in BEEF's instrumentation.

- Evaluate the final results of the POPOVER series and make recommendations on the use of BEEF as a personnel-occupied facility.

- Submit a written report of its findings to the Deputy Associate Director for Nevada Experiments and Operations.

Overview of the Review Panel Report

This report includes the following: 
- Background information and description of BEEF and POPOVER (Section 3).

- Minimum DOE explosive safety requirements (Section 4).

- POPOVER instrumentation and data (Section 5).

- Analysis of the POPOVER test series (Section 6).

- Findings and recommendations (Section 7).

- References (Section 8). 


\section{Section 3}

\section{Introduction to BEEF and POPOVER}

\section{Background Information}

$\mathrm{BEEF}$ is a facility for testing large charges of conventional $\mathrm{HE}$. The BEEF site requirements originated from Lawrence Livermore National Laboratory's B-Division Shaped Charge Scaling Project (SCSP) specifications for sophisticated diagnosis of HE charges weighing as much as several thousand pounds. Because Site 300 , the usual venue for B-Division projects, has weight limits that do not allow the testing of these large quantities of HE, an alternate test site was needed for the SCSP and other projects. After an extensive search for potential SCSP test sites, the BEEF site was chosen because it met the B-Division requirements outlined below.

The POPOVER series of HE tests was conducted to provide data for developing the technical basis to certify that BEEF is safe for personnel-occupied operations and that the structures and equipment will function as intended. Safety was the main focus of the POPOVER series. For POPOVER, BEEF was unoccupied. The maximum amount of HE detonated in the POPOVER series was 7,790 lb of TNT.

\section{Introduction to BEEF}

\section{BEEF Site Requirements}

The BEEF site meets the following B-Division requirements':

- The test site shall be within a limited-access U.S. Government facility that permits positive access control.

- The facility shall allow detonation of thousands of pounds of HE charges.

- The test site shall permit the safe remote operation of up to five nitrogen-gas-driven framing cameras and one image-converter (IC) camera housed within a buried protective bunker with an optical path not greater than $40 \mathrm{ft}(12 \mathrm{~m})$ from film plane to device center.

- The test site shall permit the safe personnel-occupied operation of two complete laser systems for IC camera and Fabry-Perot diagnostics housed within a buried protective bunker with an optical path not greater than $330 \mathrm{ft}(100 \mathrm{~m})$ from laser output to device center.

- The test site shall permit the safe personnel-occupied operation of up to 100 digitizer channels, various computers, and other control equipment housed within a buried protective bunker within a convenient distance of the test device.

- The test site shall provide shielding and protection for the Febetron radiographic units, which will be located $33 \mathrm{ft}(10 \mathrm{~m})$ from the HE charges. 
- The site shall be capable of multiple reuse with a low probability of damage to diagnostic equipment and without injury to test personnel when thousands of pounds of conventional $\mathrm{HE}$ per experiment are detonated.

\section{BEEF Site and Bunker Description}

BEEF is located in Area 4 at NTS. Two bunkers, B4-300 and B4-480, at the BEEF site were selected for the project because they met the B-Division criteria and because calculations showed that they would withstand pressures and shocks from explosive activities with minimum modifications. Figure 3-1 shows a plan view and cross section of the layout of Bunkers B4-300 and B4-480.

After verification, Bunker B4-300 will contain the personnel-occupied control room and laser sources. The BEEF bunkers will be fitted with a portable fire extinguisher system and smoke detectors. Bunker B4-480 will serve as a camera room containing five stations for high-speed framing cameras and image-converter cameras with optical ports to the firing table. A Febetron housing is located $33 \mathrm{ft}(10 \mathrm{~m})$ from, and directly in line with, the large test shots. Figure 3-2 shows the configuration of the Febetron housing before an earth berm (located just in front of the housing) was constructed.

\section{Introduction to POPOVER}

\section{POPOVER Test Objectives}

The purpose of the POPOVER test series was twofold:

1. Demonstrate that BEEF can be safely operated as a staffed facility for testing charges of thousands of pounds of $\mathrm{HE}$.

2. Demonstrate the adequacy of structures and equipment.

For the POPOVER series, BEEF was unoccupied.

The objectives of POPOVER were:

- Provide data for verifying the calculations on:

- the movement of the Febetron housing.

- the overpressure at specific locations inside and outside the bunkers.

- the relationship between atmospheric overpressure and the distance from ground zero (GZ) and the size of the explosive.

- the overall structural integrity of the bunkers to ensure against structural collapse.

- Measure the following to see if they are within acceptable limits:

- the overpressure inside the bunkers and the Febetron housings.

- accelerations in the bunkers and the Febetron housings.

- noise levels inside the personnel-occupied bunker (B4-300) and selected outside locations.

- stresses in the ceilings of the bunkers.

- stresses in the doors of the bunkers. 


\section{POPOVER Review Panel Report}
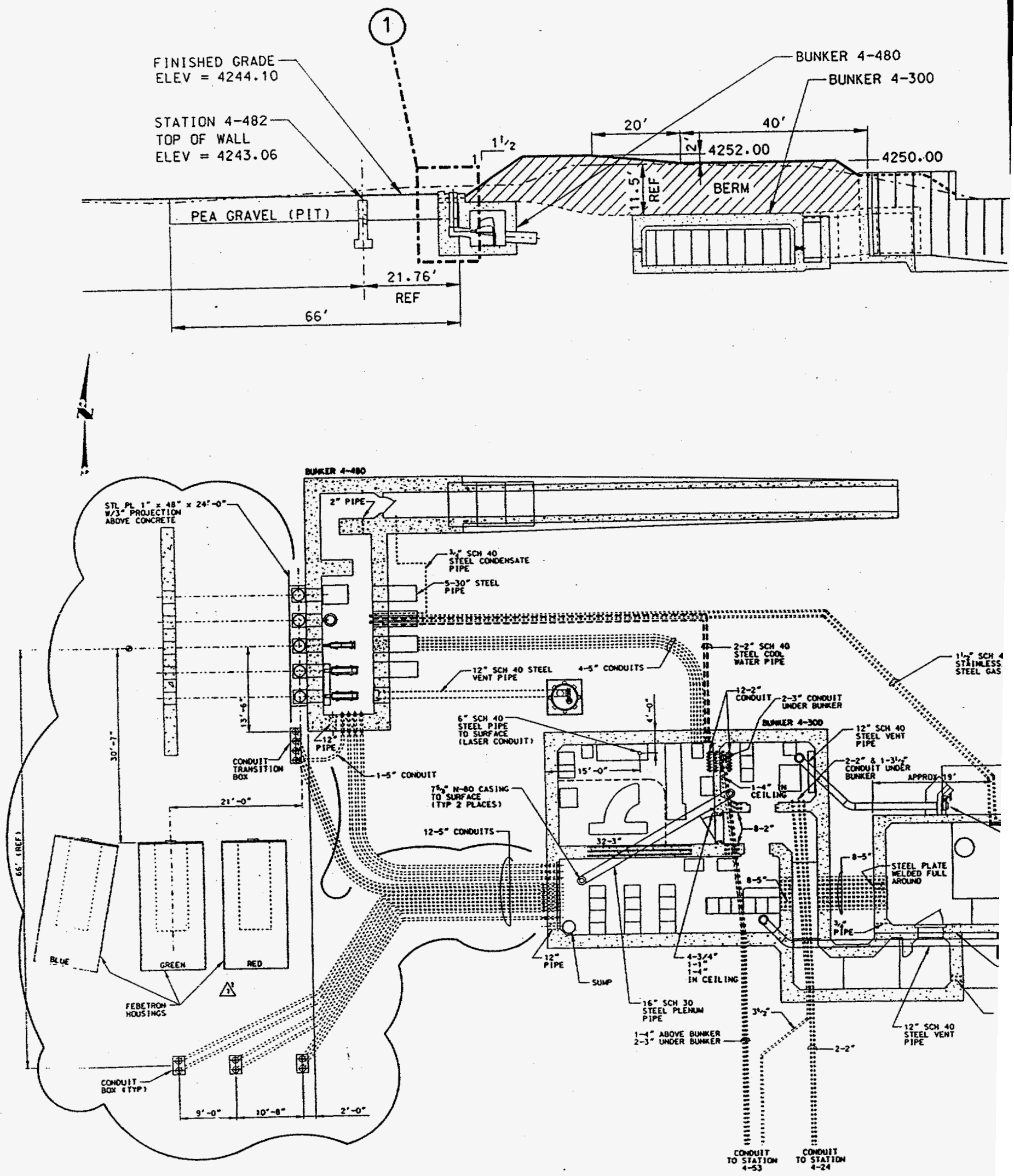

Figure 3-1. Plan view and cross section of the layout of Bunkers B4-300 and B4-480. 


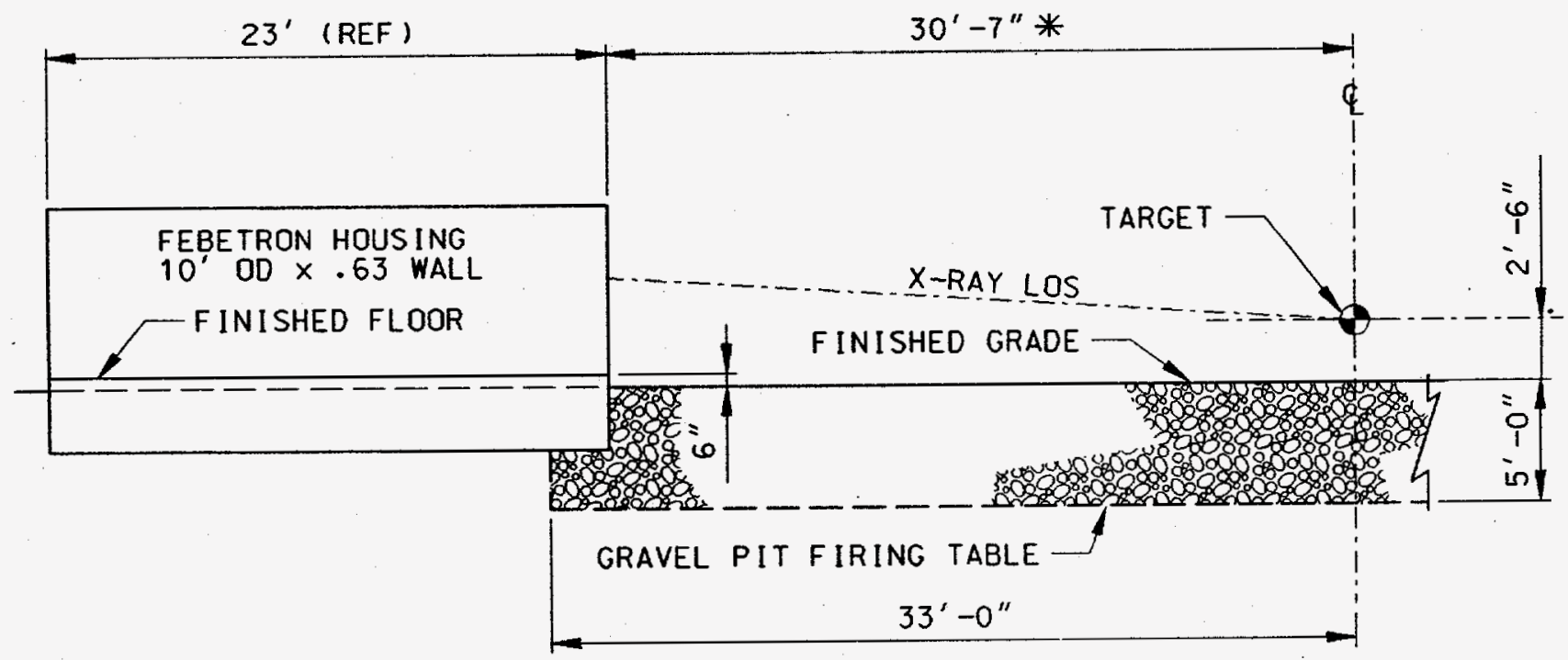

* DISTANCE FROM TARGET TO FACE OF FEBETRON HOUSING TO

PRODUCE DISTANCE OF $33^{\prime}-0$ "FROM X-RAY ANODE TO TARGET

Figure 3-2. Febetron housing configuration. 
- Monitor the effects of the explosions on the environment by video recording the plume and collecting air samples downwind of the experiment.

\section{BEEF Site and Bunker Configuration for POPOVER}

For POPOVER, the site and bunkers were in the same structural configuration as will be used in future experiments. Figure 3-1 shows the certification test configuration. This configuration allowed verification of the calculated predictions, which demonstrated that it is safe to use Bunker B4-300 as a personnel-occupied facility. It is recommended that modifications of the site or bunker configurations that might adversely affect the safe operation of the facility not be permitted without recertification and/or design review. This includes penetrations into the bunkers and removal or addition of berms inside the test area. For POPOVER, the site and the bunkers were in the following configuration:

- Site power and grounding systems installed and operating according to the provisions of the National Electrical Code (NEC).

- Firing table construction complete.

- Bunker heating, ventilation, and air conditioning (HVAC) systems installed.

- Camera high-pressure gas distribution system installed.

- All bunker penetrations (cable conduits, viewing ports, access doors, ventilation ducts, etc.) in operational condition.

- One Febetron housing (without Febetron) in position.

- Earth berm and overburden work complete.

\section{Description of the POPOVER Series}

The POPOVER test series consisted of one 512-lb diagnostic calibration test and four tests ranging in weight from 1,000 to 7,790 lb. The HE charges consisted of TNT formed into spheres, which were available in 256-lb, 1,000-lb, and 2,000-lb charges. Table 3-1 shows the HE weight and firing table position for the five tests in the POPOVER series.

POPOVER II and III consisted of single charges. POPOVER I, IV, and V each consisted of multiple charges detonated simultaneously; for POPOVER V two charges were detonated simultaneously at two different distances from Bunker B4-480. Figures 3-3 through 3-7 are schematics of the firing table layouts for the POPOVER series. 
Table 3-1. POPOVER series HE charge weights and firing table locations

\begin{tabular}{|c|c|c|}
\hline Test number & $\begin{array}{l}\text { HE charge weight } \\
\text { (Ib TNT equivalent) }\end{array}$ & $\begin{array}{l}\text { Firing table distance } \\
\text { from B4-480 (ft) }\end{array}$ \\
\hline$I$ & 512 & 15 \\
\hline II & 1,000 & 15 \\
\hline III & 1,000 & 15 \\
\hline IV & $5,022 *$ & 27 \\
\hline $\mathbf{V}$ & $\left\{\begin{array}{l}1,256 \\
6,534^{*}\end{array}\right.$ & $\begin{array}{l}15 \\
27\end{array}$ \\
\hline
\end{tabular}

*Includes $20 \mathrm{lb}$ of $\mathrm{C} 4$ (equivalent to $22.5 \mathrm{lb}$ of TNT).

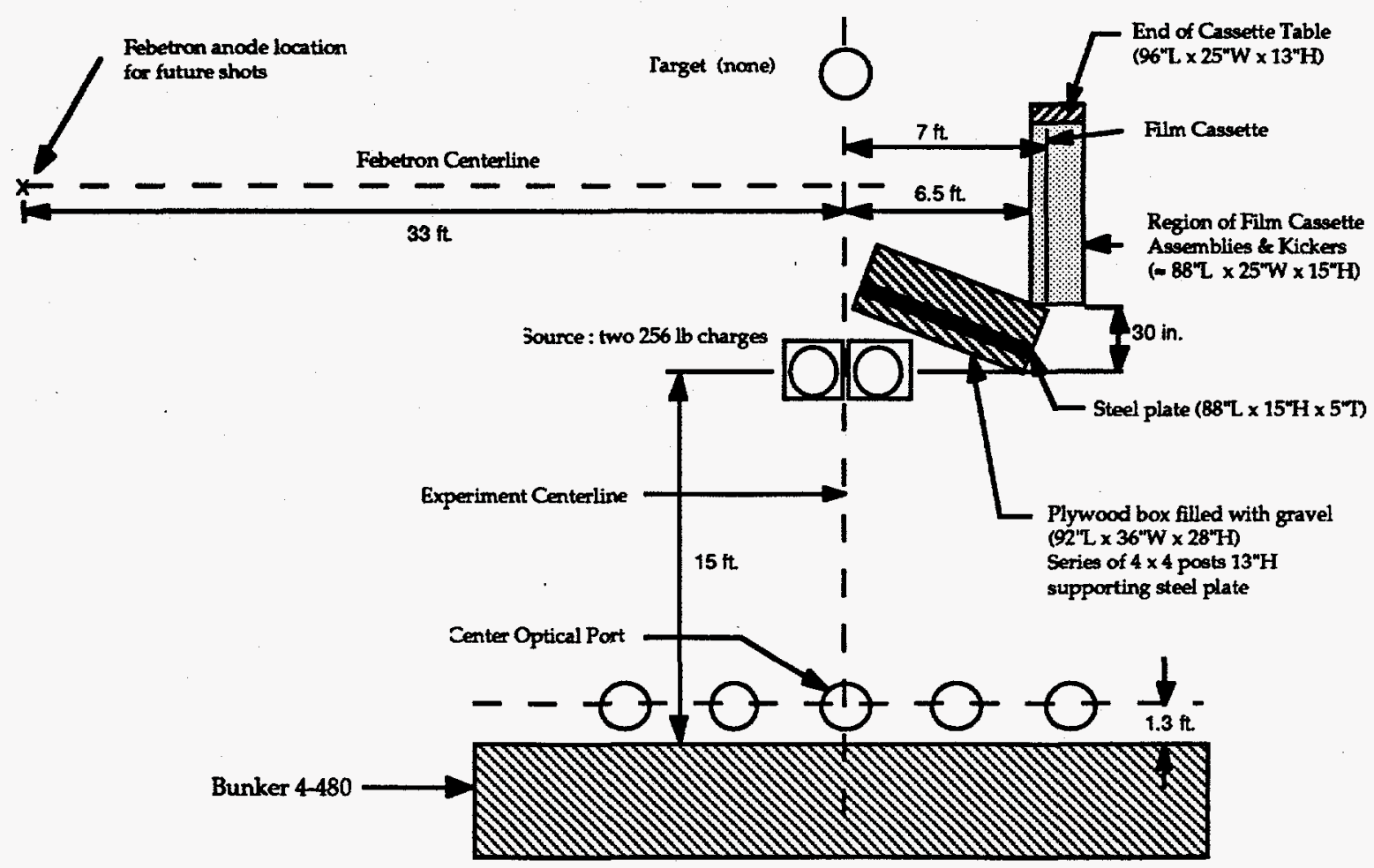

Figure 3-3. Schematic of the POPOVER I firing table layout. 


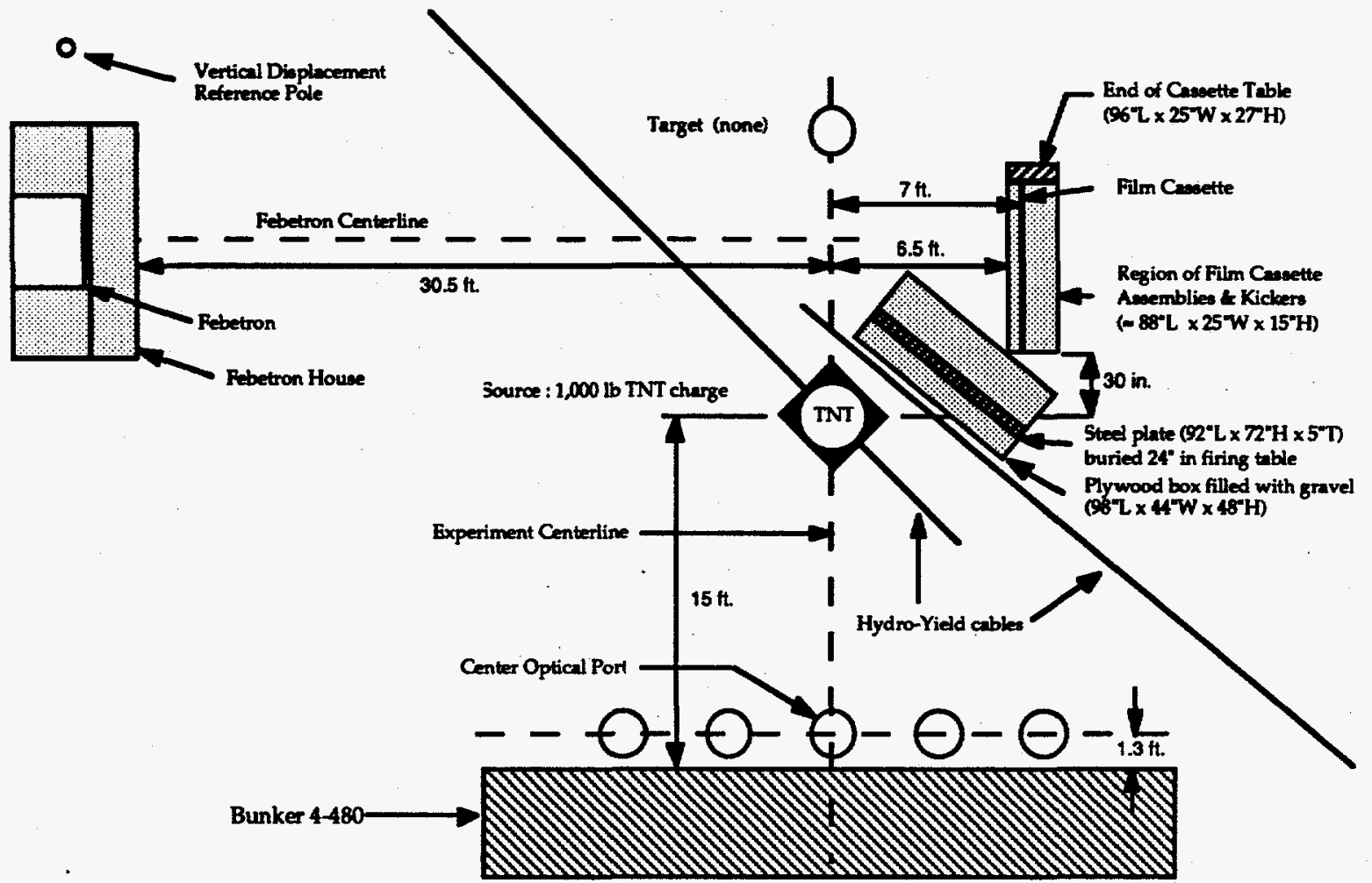

Figure 3-4. Schematic of the POPOVER II firing table layout.

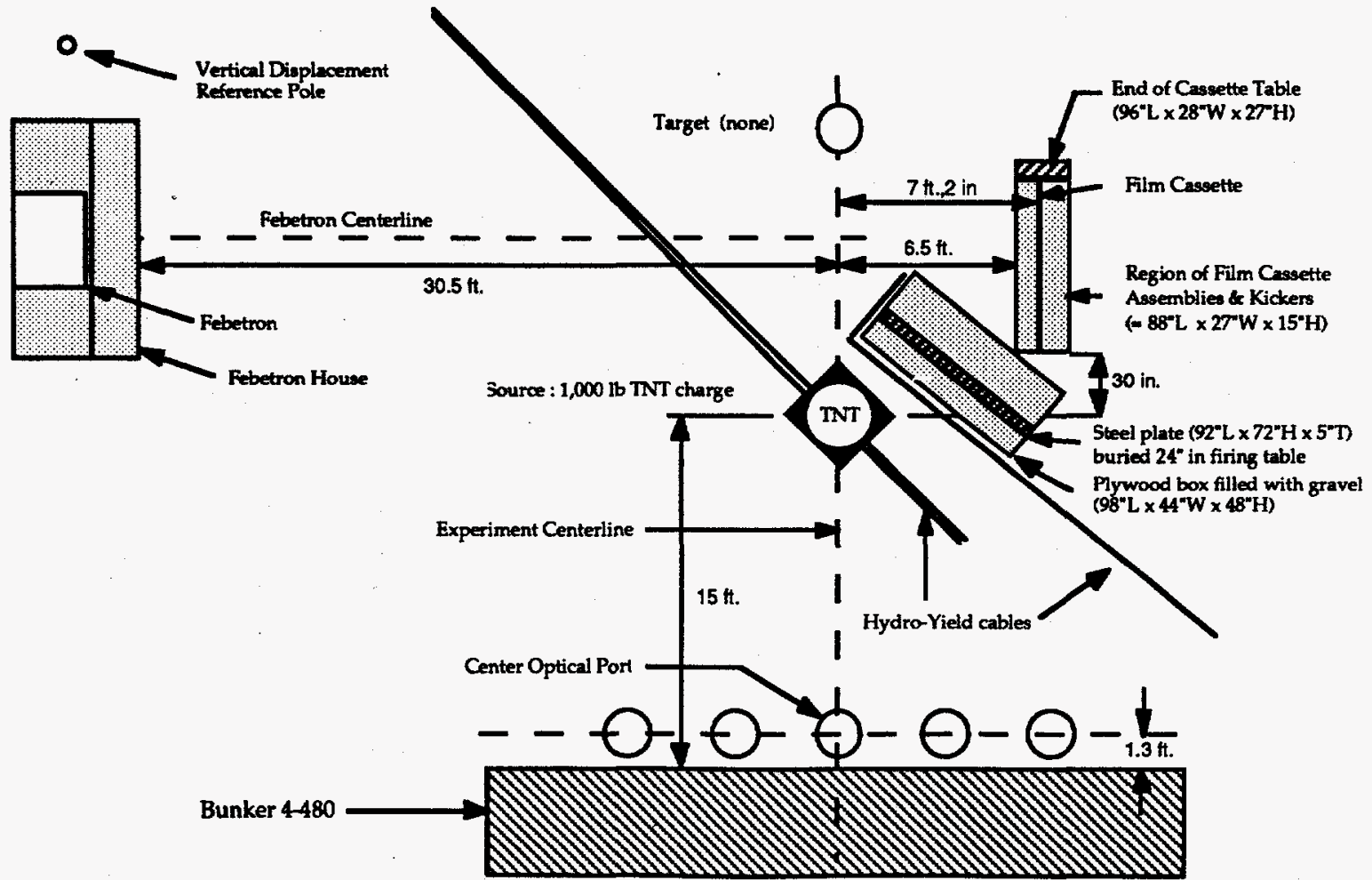

Figure 3-5. Schematic of the POPOVER III firing table layout. 


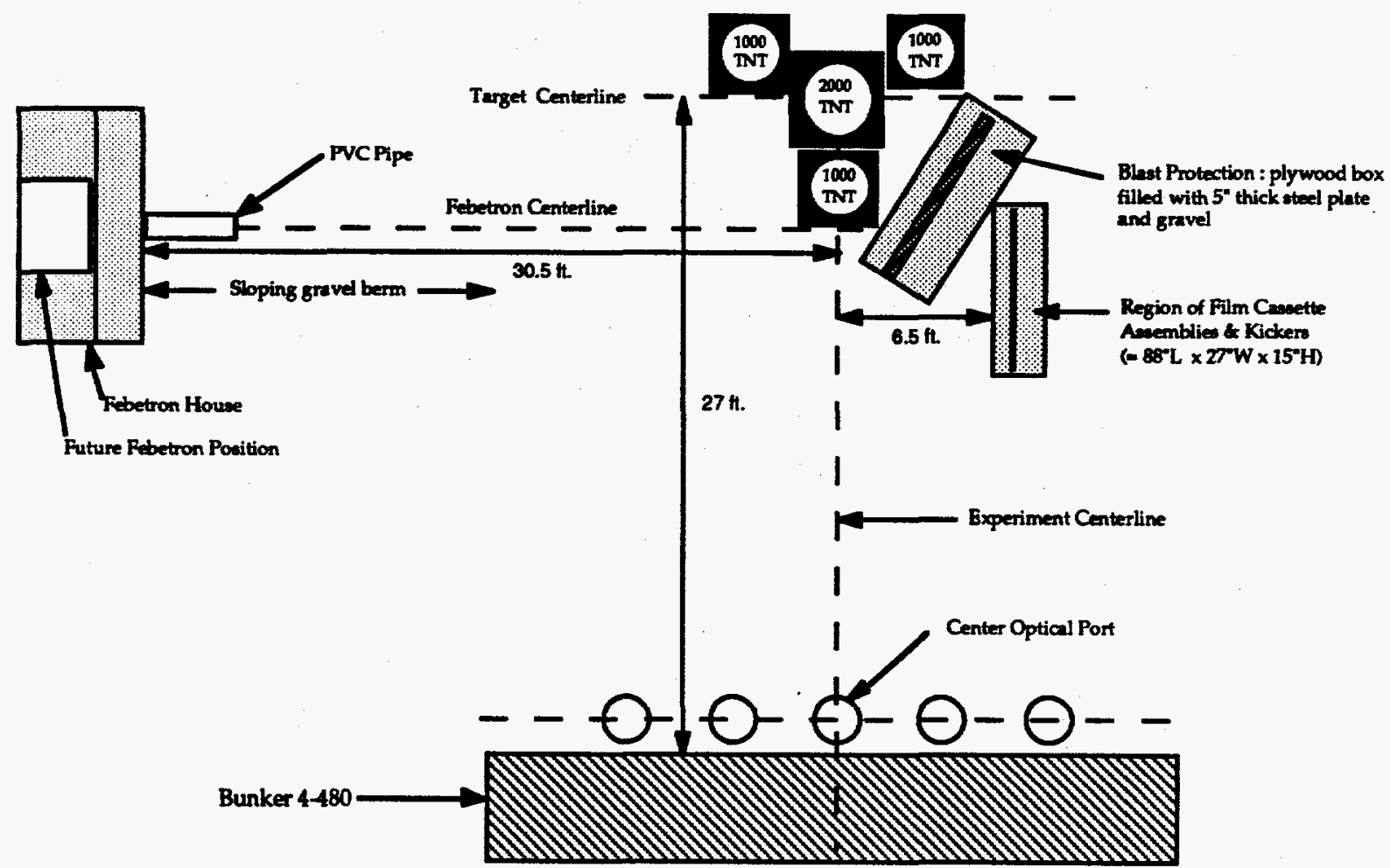

Figure 3-6. Schematic of the POPOVER IV firing table layout.

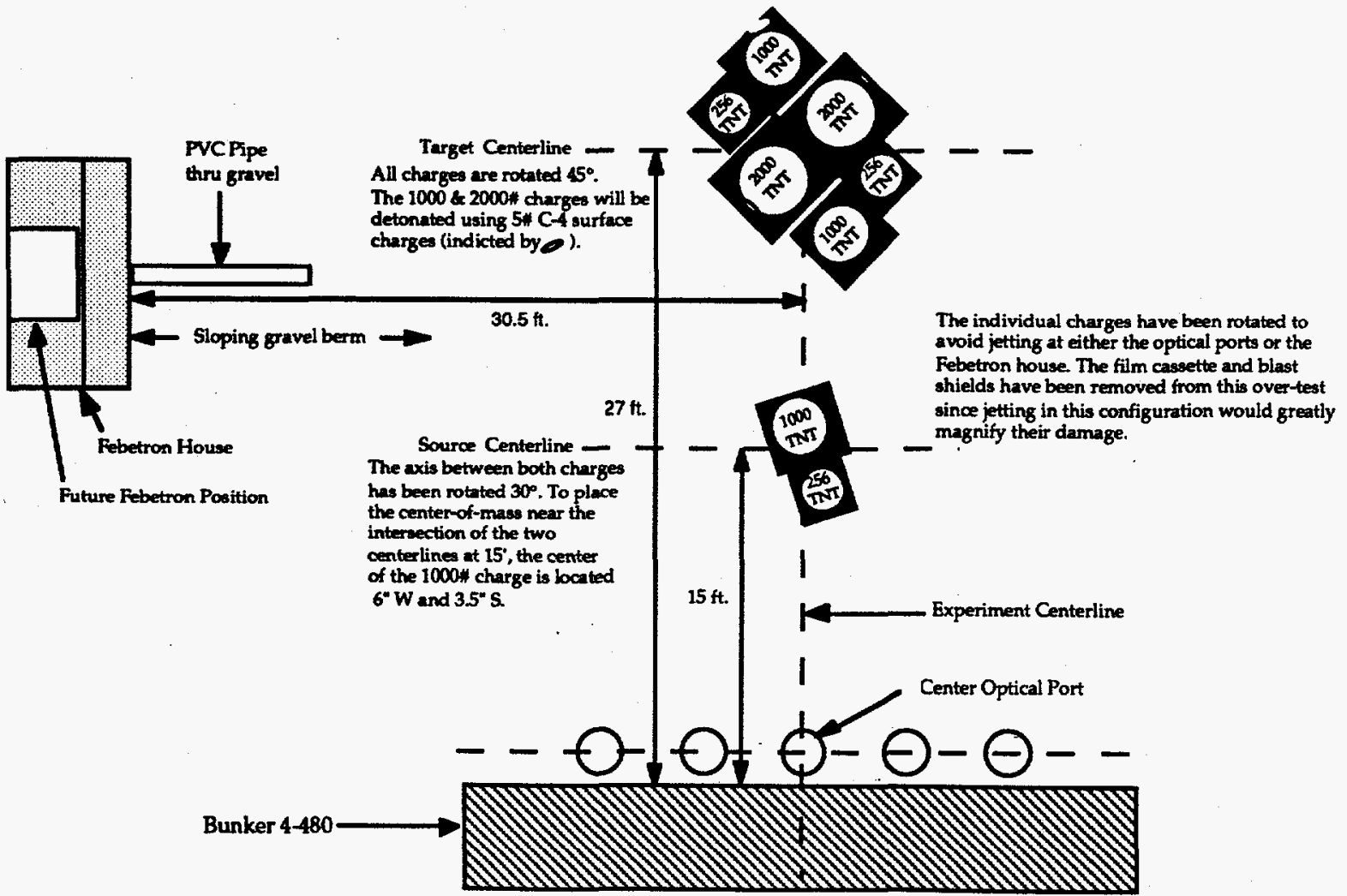

Figure 3-7. Schematic of the POPOVER V firing table layout. 
POPOVER Review Panel Report

\section{Section 4}

\section{Minimum Explosive Safety Requirements}

\section{DOE Requirements}

DOE Order 6430.1A, General Design Criteria, ${ }^{2}$ and DOE M 440.1-1, DOE Explosives Safety Manual, ${ }^{3}$ provide the minimum design and personnel protection requirements for explosive facilities. Minimum requirements from these two mandatory standards are summarized below.

\section{DOE Order 6430.1A Requirements}

Section 1307-4.2.4 of DOE Order 6430.1A states:

"Blast-resistant design for personnel and facility protection shall be based on the TNT equivalency of the maximum quantity of $\mathrm{HE}$ to be used in the bay [or at the facility]. For example, based on heat of detonation $1 \mathrm{lb}$ of one of the most energetic plastic bonded explosive is equivalent to $1.3 \mathrm{lb}$ of TNT. It is recommended by TM 5-1300 that the "effective charge weight" or the "actual charge weight," depending on the method used to determine the TNT equivalent, be increased by $20 \%$ for design purposes."

\section{DOE Explosives Safety Manual Requirements}

The DOE Explosives Safety Manual requires that each bay or facility where explosive activities are performed shall have levels of protection based on the hazard class (accident potential) determined for the activity (Chapter VI, Section 4.2). Intentional explosive detonations and other explosives activities involving a high accident potential are required to meet the criteria for DOE Hazards Class I level of protection.

Bays and facilities for Class I activities shall provide protection to prevent serious injuries to all personnel, including personnel performing the activity, personnel in other occupied areas, and all transient personnel. This protection may be achieved by controlling blast and debris through suppression, containment, or establishment of an exclusion area with positive access control. Prevention of serious injuries is satisfied where personnel will not be exposed to:

- Overpressures greater than $5 \mathrm{psig}(34 \mathrm{kPa})$ maximum effective pressure or greater than $2.3 \mathrm{psig}(16 \mathrm{kPa})$ peak positive incident pressure.

- Structural collapse of the building from overpressure or debris impact. Structural collapse is the failure of a structural component as a direct result of loss of structural 
integrity of the facility, judged according to the ability of the structure to remain intact so that explosion propagation and serious injury to personnel will not occur.

- Missiles (hazardous fragments). Hazardous fragments that can cause serious injuries are those having an impact energy greater than $11 \mathrm{ft}-\mathrm{lb}(14.9 \mathrm{~N}-\mathrm{m})$.

- Thermal fluxes greater than $0.3 \mathrm{cal} / \mathrm{cm}^{2} / \mathrm{s}\left(12.5 \mathrm{~kW} / \mathrm{m}^{2}\right)$.

The DOE minimum personnel blast protection requirement for Hazard Class I operation is equivalent to the Department of Defense permissible exposure criteria for essential personnel from intentional initiation of explosives. 4

The DOE Explosives Safety Manual also requires following DoD 6055.9-STD for quantitydistance separation requirements to protect essential personnel from being exposed to overpressures greater than $2.3 \mathrm{psig}$ ( $15.9 \mathrm{kPa}$ ), as determined by:

$$
\mathrm{K}=24 \mathrm{ft} / \mathrm{b}^{1 / 3}=\mathrm{R} / \mathrm{W}^{1 / 3} \text { for intentional detonations }
$$

where:

$\mathrm{K}=$ risk factor $\left(\mathrm{ft} / \mathrm{b} \mathrm{b}^{1 / 3}\right)$.

$\mathbf{R}=$ distance to center of detonation $(\mathrm{ft})$.

$\mathrm{W}=$ weight of explosive (lb TNT).

DoD 6055.9-STD ${ }^{4}$ also requires protection of nonessential personnel to pressures greater than $0.055 \mathrm{psig}\left(380 \mathrm{~Pa}\right.$ ) as determined by $\mathrm{K}=328 \mathrm{ft} / \mathrm{lb}{ }^{1 / 3}$ for planned detonations.

Peak overpressures are derived from the "Peak overpressure vs K-factor" nomograph in the Department of the Air Force Explosives Safety Standards. ${ }^{5}$

\section{OSHA Requirement}

In addition to the above requirements, the Occupational Safety and Health Administration (OSHA) has set an exposure limit to impulsive or impact noise of $140 \mathrm{~dB}$ peak C-weighted sound pressure, regardless of the impulse frequency or pulse duration. ${ }^{6}$ In the case of BEEF, personnel exposures to impulsive noise caused by the explosives detonation will most likely be the controlling factor. 


\section{Section 5}

\section{POPOVER Instrumentation and Data}

\section{Introduction}

EG\&G Energy Measurements, Las Vegas (EG\&G) supplied and fielded the sensors used to measure acceleration, pressure, strain, and noise intensity during the five POPOVER experiments, except that LLNL installed the strain gages and supplied four pressure sensors (P25 through P28). EG\&G was also responsible for the associated instrumentation and recording systems, as well as for the presentation of the data. LLNL was responsible for selecting the sensor types and their locations and orientations, for determining the full-scale ranges for the sensors and data channels and for the acceptance and interpretation of the data.

\section{Documentation of Sensors and Instruments}

EG\& $G$ has released an engineering report ${ }^{7}$ that documents the traceability of the data from the sensors to the recording media and from the recording media to engineering units. The EG\&G report is an essential reference used to produce this report. Its contents are as follows:

\begin{tabular}{|lc|}
\hline \multicolumn{1}{|c|}{ Subject } & Section \\
\hline Final Engineering Report & 1 \\
Overview & \\
Facilities & \\
Personnel & \\
Power and Grounding & \\
Measurement Summary & \\
Measurement Descriptions & \\
Blast Overpressure & \\
Acceleration - wide band & \\
Acceleration - low level & \\
Velocity & \\
Strain & $\mathbf{2}$ \\
Other Measurements & $\mathbf{3}$ \\
Fielding Effort & \\
Pre-Fielding Preparations & \\
POPOVER-1 through -5 & \\
Measurement Cabling, Grounding, and Shielding & \\
POPOVER Instrumentation Emplacement & \\
EG\&G Drawing EJG94-201140-0B & \\
\end{tabular}




\begin{tabular}{|lc|}
\hline \multicolumn{1}{|c|}{ Subject } & Section \\
\hline Final Physics/Instrumentation Package POPOVER-1 through -5 & 4 \\
Sensor locations, data channel ID, sensors' manufacturer and model, & \\
sensor serial numbers, predicted data ranges, system data ranges, full & \\
scales of amplified signals, calibration signal levels, and recording \\
devices used (MERS is Multiple Event Recording System digitizer, & \\
TRAQH is Transient Acquisition High speed digitizer) & \\
Transducer Calibration POPOVER-1 through -5 & 5 \\
PCB pressure sensors & \\
JP Technologies strain gages & \\
Paroscientific Digiquartz pressure sensor & \\
Endevco accelerometers & \\
Sundstrand accelerometers & \\
Identifications and calibrations of added or replaced sensors & 6 \\
Trailer Log & 7 \\
Channel History & $\mathbf{8}$ \\
Transducer Checkout Logs & 9 \\
Event Checklists POPOVER-1 through -5 & 10 \\
Timing \& Control Support Requirements & 11 \\
Construction \& Engineering Support Requirements & 12 \\
Cable Criteria & \\
One-Lines POPOVER-1 through -5 & \\
Overview schematics of Fidu and timing signals, sensors, sensor \\
power supplies, signal conditioners, filters and recording systems \\
(analog tape and digital solid state recorders) & 13 \\
PCB Channel Calibrator & \\
Description, calibration, operational procedures, and schematic of a & \\
custom piezo-electric sensor simulator & 14 \\
MERS Engineering Units Conversion Table & 16 \\
Photos & \\
Transducer Information & \\
\hline
\end{tabular}

Schematics identifying the location of the instrumentation for each of the POPOVER tests are given in Figs. 5-1 though 5-5. The final data from these instruments is given in a separate document. ${ }^{8}$ Tables 5-1 through 5-5 give the predicted peak values, the preliminary data (which were used to make the decision to continue with the next POPOVER test), the final data, and the associated final test comments. Attempts have been made to identify and to exclude from the tables those peak values resulting from extraneous electrical and mechanical noise. The tabulated peak audio noise levels have been collected from static readings. 


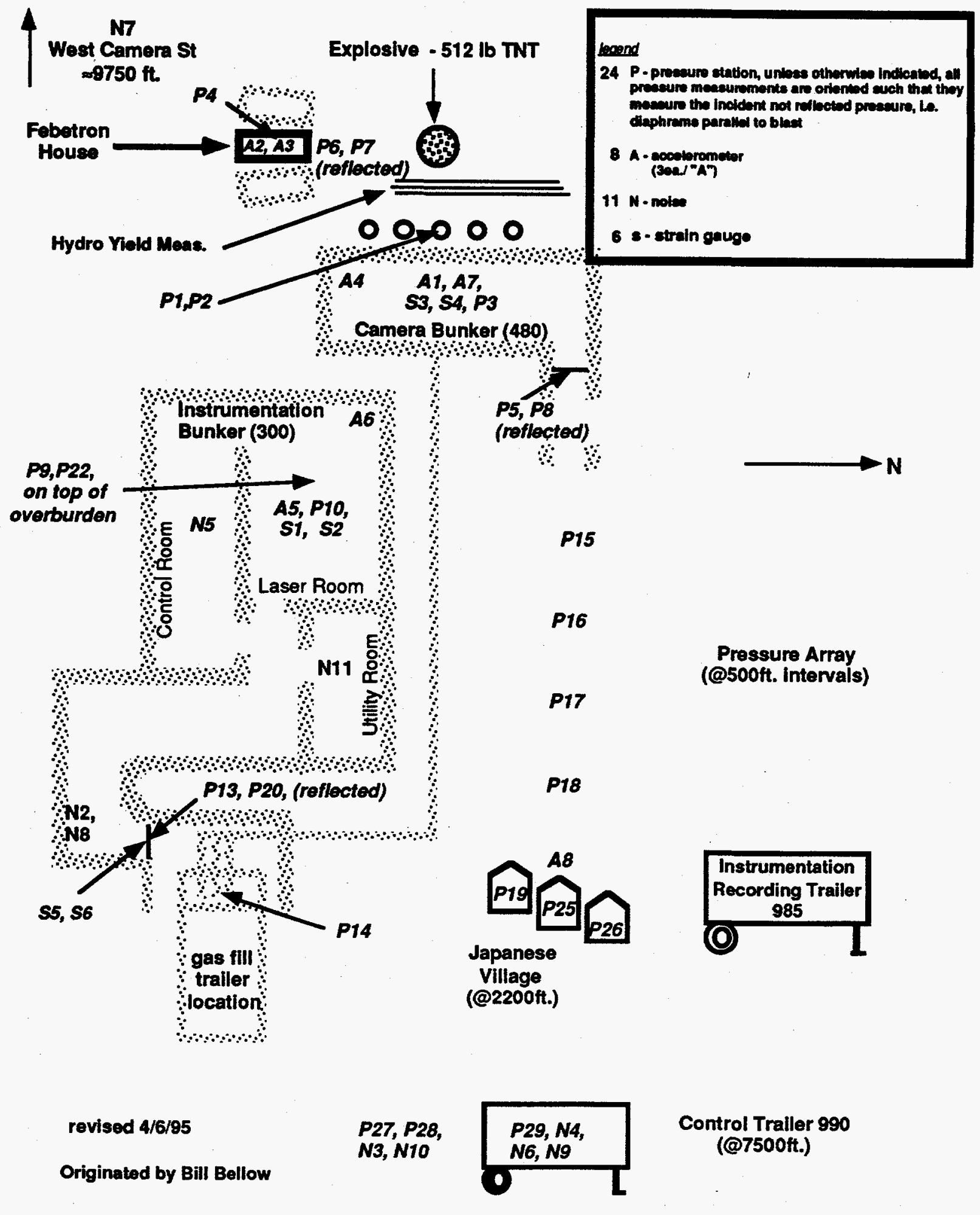

Figure 5-1. Location of instrumentation for the POPOVER I test. 


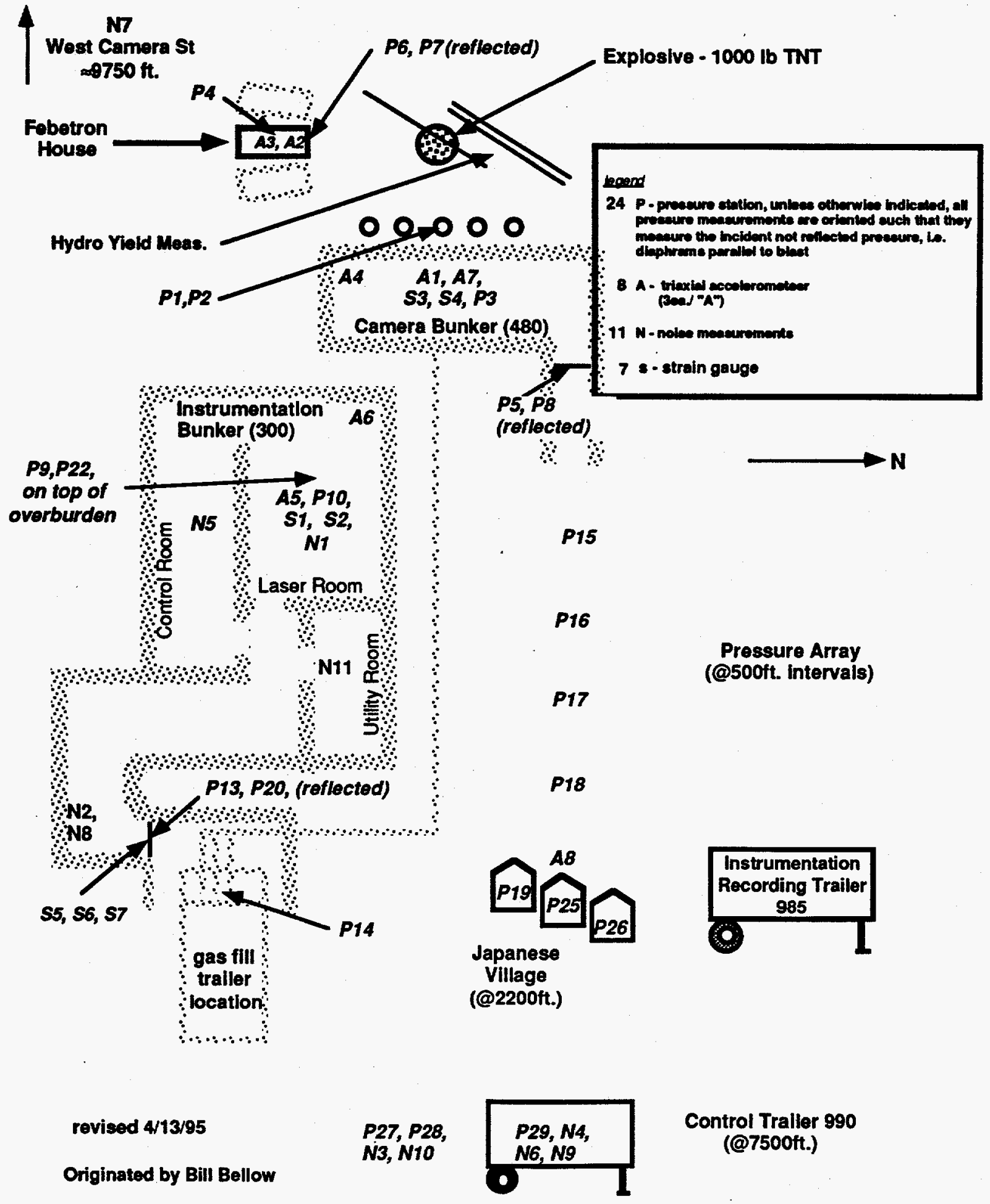

Figure 5-2. Location of instrumentation for the POPOVER II test. 


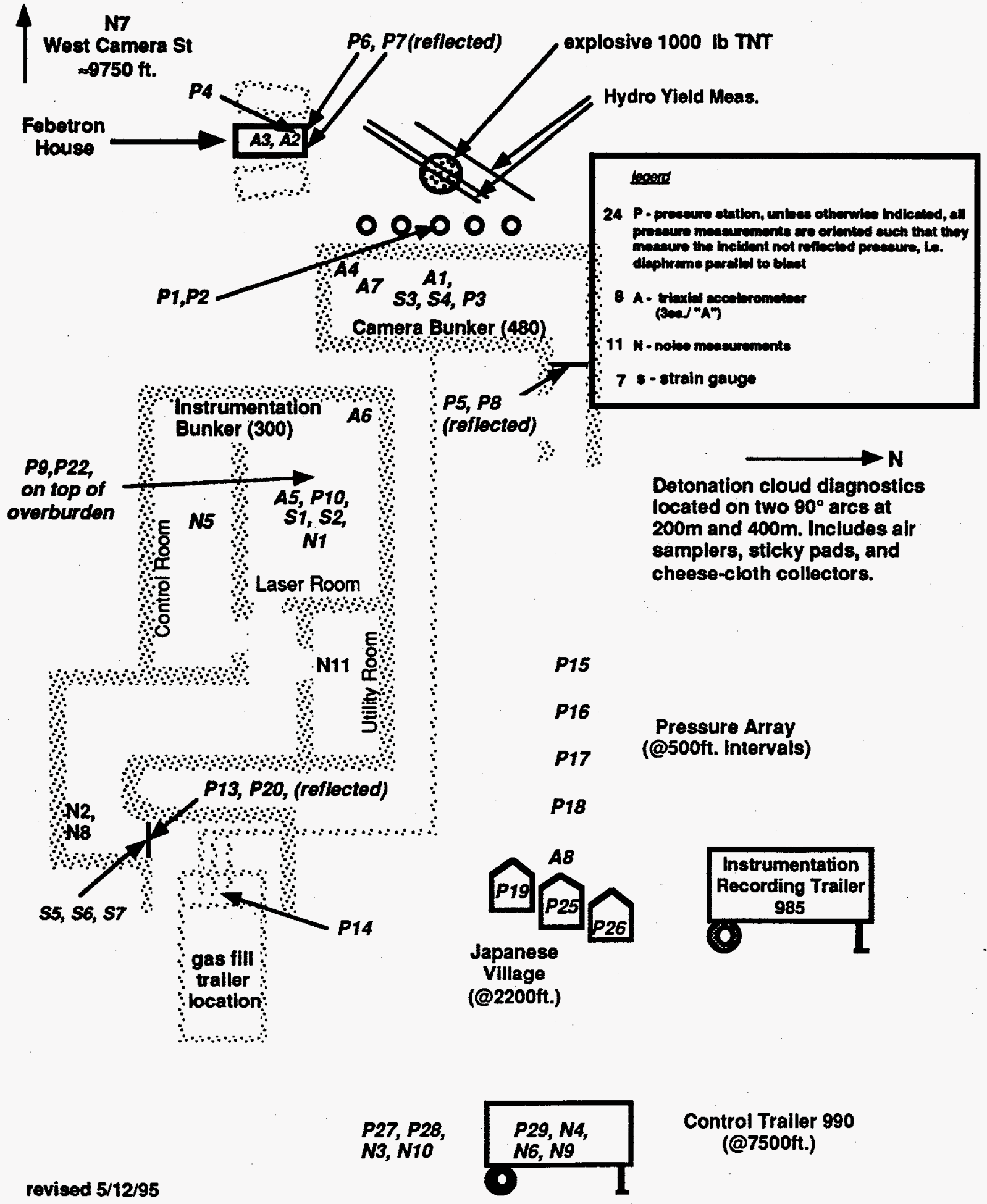

Figure 5-3. Location of instrumentation for the POPOVER III test. 


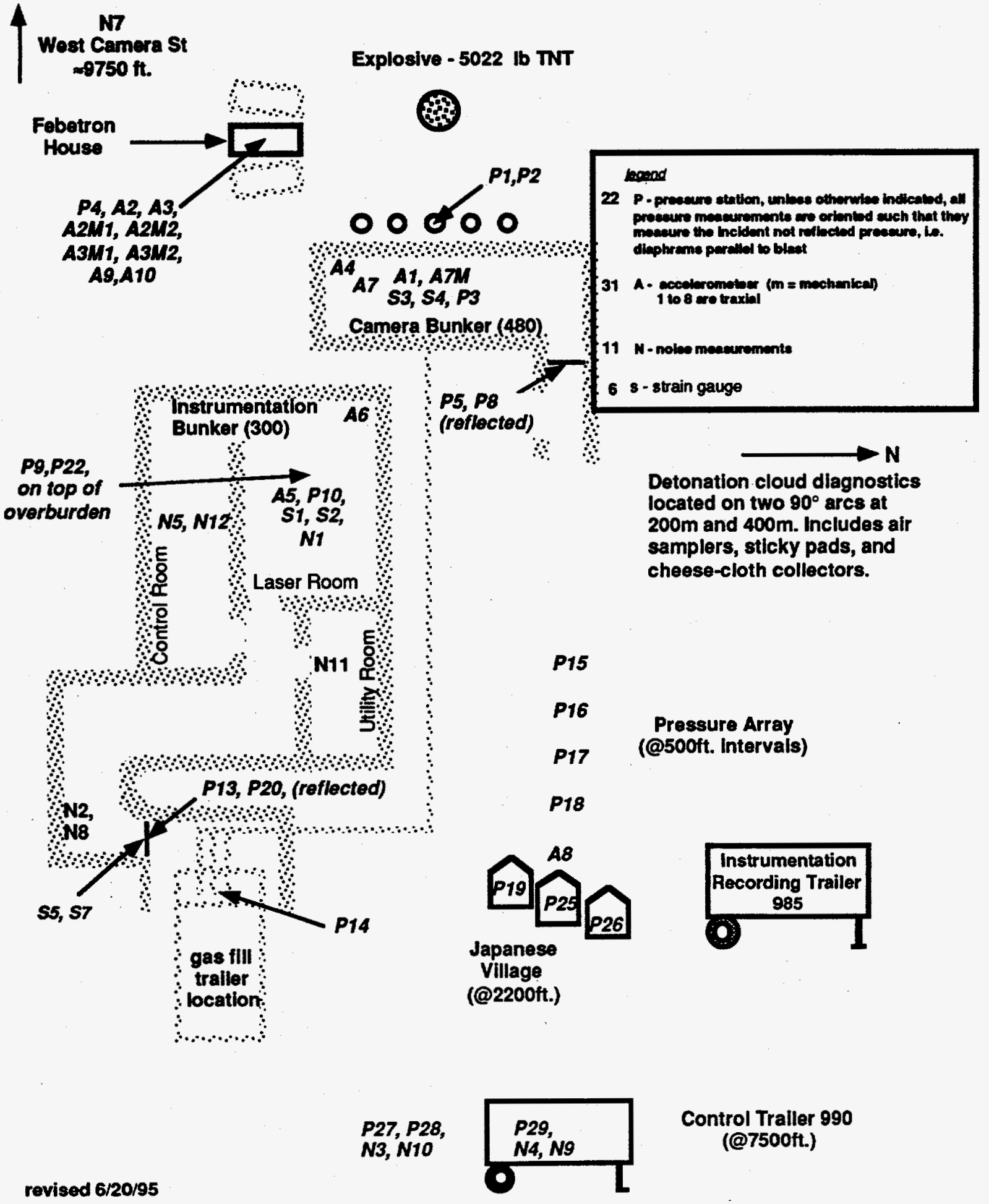

Figure 5-4. Location of instrumentation for the POPOVER IV test. 


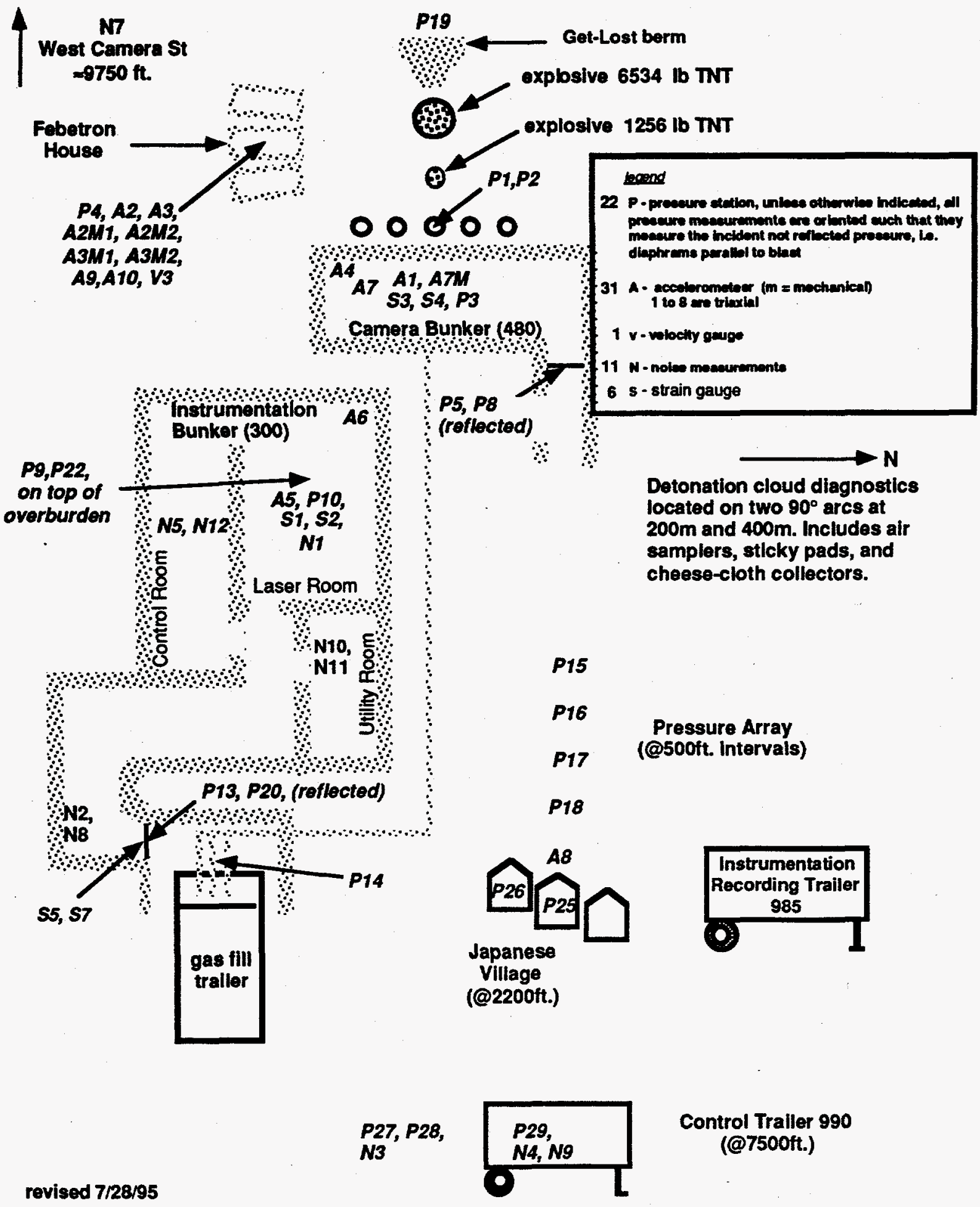

Figure 5-5. Location of instrumentation for the POPOVER $V$ test. 
Table 5-1. Peak pressures, strains, and accelerations in POPOVER I

Units of the datu wre: proware, prigfetrain, $10^{-6}$; sccelention, g; scund intensity, dBC. NS = no sensor. DEF = defective sensor, cable, or instrument.

\begin{tabular}{|c|c|c|c|c|c|}
\hline Seneor & Lacention & $\begin{array}{l}\text { Predicted } \\
\text { peak } \\
\text { magnitude }\end{array}$ & $\begin{array}{l}\text { Preliminary } \\
\text { peak } \\
\text { magnitude }\end{array}$ & $\begin{array}{l}\text { Final } \\
\text { peak } \\
\text { moenltude }\end{array}$ & Comments on final date \\
\hline $\begin{array}{l}\text { P1 } \\
\text { P2 } \\
\text { P5 } \\
\text { P8 } \\
\text { P3 }\end{array}$ & $\begin{array}{l}\text { Outside B4-480 } \\
\text { Outride B4-480 } \\
\text { Outride B4-480 door } \\
\text { Outside B4-480 door } \\
\text { Invide B4-480 }\end{array}$ & $\begin{array}{l}440 \\
440 \\
<44 \\
<44 \\
0\end{array}$ & $\begin{array}{c}7960 \\
520 \\
7.1 \\
7.7 \\
?\end{array}$ & $\begin{array}{l}7060 \\
520 \\
8.1 \\
7.7 \\
0.06\end{array}$ & \\
\hline $\begin{array}{l}\text { P6 } \\
\text { P7 } \\
\text { P4 } \\
\text { P22 }\end{array}$ & $\begin{array}{l}\text { Front cutride Fobetron } \\
\text { Front ourside Febetron } \\
\text { Invide Fobetron } \\
\text { Outride top B4-300 } \\
\text { Outride top B4-300 }\end{array}$ & $\begin{array}{l}15 \\
15\end{array}$ & $\begin{array}{l}13.6 \\
11.2\end{array}$ & $\begin{array}{l}\text { NS } \\
\text { NS } \\
13.8 \\
12.3\end{array}$ & \\
\hline $\begin{array}{l}\text { P10 } \\
\text { P13 } \\
\text { P20 } \\
\text { P14 } \\
\text { P15 }\end{array}$ & $\begin{array}{l}\text { Invide B4-300 } \\
\text { Outride docr B4-300 } \\
\text { Outride door B4-300 } \\
\text { Near N2 triler } \\
\text { Spaced amy \& 500' }\end{array}$ & $\begin{array}{l}0 \\
<3.8 \\
<3.8 \\
<3.2 \\
<1\end{array}$ & $\begin{array}{c}-0.16 \\
2.75 \\
2 \\
1.5 \\
0.58\end{array}$ & $\begin{array}{l}20.05 \\
3.7 \\
2 \\
\text { DEF } \\
0.6\end{array}$ & Noiee? \\
\hline $\begin{array}{l}\text { P16 } \\
\text { P17 } \\
\text { P18 } \\
\text { P19 } \\
\text { P25 }\end{array}$ & $\begin{array}{l}\text { Spaced array \& } 1000^{\circ} \\
\text { Spaced array } 1500^{\circ} \\
\text { Spaced arry } 2000^{\circ} \\
\text { Japanece Village } 92126 \\
\text { Iepancie Villyge } 2109^{\prime}\end{array}$ & $\begin{array}{l}<0.7 \\
<0.4 \\
<0.1 \\
<.1 \\
<0.1\end{array}$ & $\begin{array}{l}0.13 \\
0.11 \\
0.09 \\
0.07 \\
0.05\end{array}$ & $\begin{array}{l}0.15 \\
0.12 \\
0.1 \\
0.07 \\
0.05 \\
\end{array}$ & \\
\hline $\begin{array}{l}\text { P26 } \\
\text { P27 } \\
\text { P28 } \\
\text { P19 } \\
\text { P26 }\end{array}$ & $\begin{array}{l}\text { Japanewe Village } \Theta 2314^{\prime} \\
\text { Outcide control trailer } 900{ }^{\prime} \\
\text { Outside control trailer } 9 \text { 7468' } \\
\text { Behind get-lost berm } \\
\text { Behind Mt. Winer }\end{array}$ & $\begin{array}{l}40.1 \\
6.021 \\
\$ .021\end{array}$ & 0.06 & $\begin{array}{l}0.06 \\
0.02 \\
\text { DEF } \\
\text { NS } \\
\end{array}$ & \\
\hline $\begin{array}{l}\text { P29 } \\
\text { S1 } \\
\text { S2 } \\
\text { S3 } \\
\text { S4 } \\
\end{array}$ & $\begin{array}{l}\text { Inside control trailer @ } 7500 \\
\text { Celing B4-300 } \\
\text { Ceiling B4-300 } \\
\text { Ceiling B4-480 } \\
\text { Ceiling B4-480 }\end{array}$ & $\begin{array}{c}0 \\
242 \\
242 \\
1211 \\
1211 \\
\end{array}$ & $\begin{array}{l}-235 /-10 \\
-135 /-10 \\
-440 / 280 \\
-430 / 275 \\
\end{array}$ & $\begin{array}{l}\text { DEF } \\
-30 / 20 \\
-20 / 20 \\
-7 / 350 \\
-7 / 320\end{array}$ & $\begin{array}{l}\text { Spikes in first } 16 \mathrm{msec} \text { are considered noive } \\
\text { Spikes in first } 16 \mathrm{msec} \text { are considered noise } \\
\text { Spikes in first } 16 \mathrm{msec} \text { are considered noise } \\
\text { Spikes in first } 16 \mathrm{msec} \text { are considered noise }\end{array}$ \\
\hline $\begin{array}{l}\text { S5 } \\
\text { S6 } \\
\text { S7 } \\
\text { A1V } \\
\text { AlR }\end{array}$ & $\begin{array}{l}\text { Door B4-300 } \\
\text { Door B4-300 } \\
\text { Door bolt B4-300 } \\
\text { B4-480: center of floor } \\
\text { B4-480: center of floor }\end{array}$ & $\begin{array}{l}125 \\
125 \\
3.2 \\
3.2\end{array}$ & $\begin{array}{c}-125 / \sim 10 \\
-124 / \sim 10 \\
>5 \\
>5\end{array}$ & $\begin{array}{l}-26 / 30 \\
-25 / 26 \\
N 5 \\
>5 \\
>5\end{array}$ & $\begin{array}{l}\text { Spikes in first } 16 \mathrm{msec} \text { are considered noise } \\
\text { Spikes in first } 16 \mathrm{msec} \text { are considered noise }\end{array}$ \\
\hline $\begin{array}{l}\text { A1T } \\
\text { A4V } \\
\text { A4R } \\
\text { A4T } \\
\text { ATV }\end{array}$ & $\begin{array}{l}\text { B4-480: center of floor } \\
\text { B4-480: SW comer of floor } \\
\text { B4-480: SW comer of floor } \\
\text { B4-480: SW comer of floor } \\
\text { B4-480: dummy camers. }\end{array}$ & $\begin{array}{l}3.2 \\
3.2 \\
3.2 \\
3.2\end{array}$ & $\begin{array}{l}>5 \\
>5 \\
>5 \\
>5 \\
>5\end{array}$ & $\begin{array}{l}>5 \\
>5 \\
>5 \\
>5 \\
>5\end{array}$ & \\
\hline $\begin{array}{l}\text { ATR } \\
\text { ATT } \\
\text { A2V } \\
\text { A2RT }\end{array}$ & $\begin{array}{l}\text { B4-480: dummy cemera } \\
\text { B4-480, dummy ctmer } \\
\text { Febetron housing: NW comer } \\
\text { Febetron housing: NW comer } \\
\text { Febetron housing: NW comer. }\end{array}$ & $\begin{array}{l}1.1 \\
1.1 \\
1.1\end{array}$ & $\begin{array}{l}>5 \\
4.1\end{array}$ & $\begin{array}{l}75 \\
4 \\
\mathrm{NS} \\
\mathrm{N}\end{array}$ & . \\
\hline $\begin{array}{l}\text { A3V } \\
\text { A3R } \\
\text { A3T } \\
\text { A9R } \\
\text { A10T }\end{array}$ & $\begin{array}{l}\text { Febetron housing: cemer of floce } \\
\text { Febetron housing; center of floce } \\
\text { Febetron housing: center of floor } \\
\text { Feberron housing: inside bulthead } \\
\text { Febetron housing: bottle reck }\end{array}$ & $\begin{array}{l}0.6 \\
0.6 \\
0.6\end{array}$ & & $\begin{array}{l}\mathbf{N} \\
\mathbf{N} \\
\mathbf{N} \\
\mathrm{N} \\
\mathrm{N}\end{array}$ & \\
\hline $\begin{array}{l}\text { ASV } \\
\text { ASR } \\
\text { AST } \\
\text { AGV } \\
\text { AGR }\end{array}$ & $\begin{array}{l}\text { B4-300: center of laser roam flocr } \\
\text { B4-300: center of laser room floor } \\
\text { B4-300: center of laser room floor } \\
\text { B4-300: NW comer of laser room floor } \\
\text { B4-300: NW comer of laser room floor }\end{array}$ & $\begin{array}{l}0.2 \\
0.2 \\
0.2 \\
0.3 \\
0.3 \\
\end{array}$ & $\begin{array}{r}0.44 \\
0.14 \\
0.06 \\
0.2 \\
0.09\end{array}$ & $\begin{array}{l}0.41 \\
0.13 \\
0.06 \\
0.19 \\
0.09\end{array}$ & \\
\hline $\begin{array}{l}\text { A6T } \\
\text { A8V } \\
\text { A8R } \\
\text { A8T } \\
\text { N1-A }\end{array}$ & $\begin{array}{l}\text { B4-300: NW comer of laser room floor } \\
\text { Japanese Village @2105 } \\
\text { Japanese Village @ } 2105 \\
\text { Japanese Village } 2105 \\
\text { Inside B4-300 loser room }\end{array}$ & $\begin{array}{l}0.3 \\
<0.1 \\
<0.1 \\
<0.1 \\
-120\end{array}$ & 109 & $\begin{array}{l}0.05 \\
0.06 \\
0.06 \\
0.023 \\
109 \\
\end{array}$ & \\
\hline $\begin{array}{l}\text { N1-B } \\
\text { N1-C (peak) } \\
\text { NS-A } \\
\text { N5-B } \\
\text { N12 (peak) }\end{array}$ & $\begin{array}{l}\text { Inside B4-300 laser room } \\
\text { Inside B4-300 laser room } \\
\text { Inside B4-300 control room } \\
\text { Inside B4-300 control room } \\
\text { Inside B4-300 control room }\end{array}$ & -120 & $\begin{array}{l}\mathbf{N S} \\
\mathbf{N S} \\
\mathbf{1 1 4} \\
\mathbf{N S} \\
\mathbf{N S}\end{array}$ & $\begin{array}{l}\text { NS } \\
\text { NS } \\
114 \\
\text { NS } \\
\text { NS }\end{array}$ & \\
\hline $\begin{array}{l}\text { N10-A } \\
\text { N10-B } \\
\text { N11-A (peak) } \\
\text { N11-B (peak) } \\
\text { N2-A }\end{array}$ & $\begin{array}{l}\text { Inside B4-300 utility room } \\
\text { Inside B4-300 ulitity room } \\
\text { Inside B4-300 utility room } \\
\text { Inside B4-300 utility room } \\
\text { Entrance door B4-300 }\end{array}$ & $\begin{array}{r}-120 \\
-120 \\
\end{array}$ & $\begin{array}{l}\text { NS } \\
\text { NS } \\
\text { 125 } \\
\text { NS } \\
\text { DEF }\end{array}$ & $\begin{array}{l}\text { N } \\
\text { IS } \\
\text { NS } \\
\text { DEF }\end{array}$ & \\
\hline $\begin{array}{l}\text { N2-B } \\
\text { N8-A (peak) } \\
\text { N8-B (peak) } \\
\text { N3 (peak) } \\
\text { N10-A }\end{array}$ & $\begin{array}{l}\text { Entrance door B4-300 } \\
\text { Entrance door B4-300 } \\
\text { Entrance door B4-300 } \\
\text { Outride control trailer } \\
\text { Outside control trailer }\end{array}$ & $\begin{array}{l}-120 \\
<140 \\
<140\end{array}$ & $\begin{array}{l}\text { NS } \\
128 \\
\text { N } \\
125 \\
128\end{array}$ & $\begin{array}{l}\mathbf{N} \\
128 \\
\mathbf{N} \\
125 \\
128\end{array}$ & \\
\hline $\begin{array}{l}\text { N10-B } \\
\text { N4-A } \\
\text { N4B } \\
\text { N6 } \\
\text { N9 (peak) } \\
N 7 \text { (peak) }\end{array}$ & $\begin{array}{l}\text { Ortside control trailer } \\
\text { Inside control trailer } \\
\text { Inside control trailer } \\
\text { Inside control trailer } \\
\text { Inside control trailer } \\
\text { Camera station } \$ 7(9750)\end{array}$ & $\begin{array}{l}<140 \\
<140 \\
<140 \\
<140\end{array}$ & $\begin{array}{l}\mathbf{N} \\
120 \\
\mathbf{N} \\
127 \\
131 \\
127\end{array}$ & $\begin{array}{l}\mathbf{N} \\
120 \\
\mathbf{N} \\
127 \\
131 \\
127\end{array}$ & . \\
\hline
\end{tabular}


Table 5-2. Peak pressures, strains, and accelerations in POPOVER II

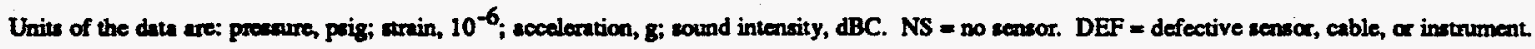

\begin{tabular}{|c|c|c|c|c|c|}
\hline Sensor & Location & $\begin{array}{l}\text { Predicted } \\
\text { peak } \\
\text { marnitude }\end{array}$ & $\begin{array}{l}\text { Prelliminary } \\
\text { peak } \\
\text { magnitude }\end{array}$ & $\begin{array}{l}\text { Final } \\
\text { peak } \\
\text { marnitude }\end{array}$ & Comments on hnal data \\
\hline $\begin{array}{l}P_{1} \\
P_{2} \\
P_{3} \\
P_{8} \\
P_{3}\end{array}$ & $\begin{array}{l}\text { Ourvide B4-480 } \\
\text { Cutride B4-480 } \\
\text { Ourride B4-480 door } \\
\text { Oureide B4480 door } \\
\text { Inside B4-480 }\end{array}$ & $\begin{array}{c}640 \\
640 \\
44 \\
44 \\
0\end{array}$ & $\begin{array}{c}650 \\
620 \\
-6 / 12 \\
-6.5 / 12 \\
0.03 \\
\end{array}$ & $\begin{array}{l}770 \\
680 \\
12 \\
12 \\
0.02 \\
\end{array}$ & \\
\hline $\begin{array}{l}\text { P6 } \\
\text { P7 } \\
\text { P4 } \\
\text { P9 } \\
\text { P22 }\end{array}$ & $\begin{array}{l}\text { Front outride Febetron } \\
\text { Front outside Fotetron } \\
\text { Inside Febetron } \\
\text { Outride top B4300 } \\
\text { Outride top B4-300 }\end{array}$ & $\begin{array}{c}454 \\
454 \\
0 \\
24.5 \\
24.5 \\
\end{array}$ & $\begin{array}{c}1000 \\
0.37 \\
24 \\
17 \\
\end{array}$ & $\begin{array}{l}>900 \\
\mathrm{DEF} \\
>0.5 \\
25 \\
17 \\
\end{array}$ & Struck by debris? \\
\hline $\begin{array}{l}\text { P10 } \\
\text { P13 } \\
\text { P20 } \\
\text { P14 } \\
\text { P15 }\end{array}$ & $\begin{array}{l}\text { Inside B4300 } \\
\text { Outride door B4-300 } \\
\text { Outside doc B4-300 } \\
\text { Near N2 trailer } 500 \\
\text { Speced amay } 900\end{array}$ & $\begin{array}{c}0 \\
3.8 \\
3.8 \\
3.2 \\
1\end{array}$ & $\begin{array}{l}0.01 \\
4.4 \\
4.7 \\
3.0 \\
0.86 \\
\end{array}$ & $\begin{array}{l}0.008 \\
4.3 \\
4.6 \\
1.5 \\
0.87 \\
\end{array}$ & \\
\hline $\begin{array}{l}\text { P16 } \\
\text { P17 } \\
\text { P18 } \\
\text { P19 } \\
\text { P25 }\end{array}$ & 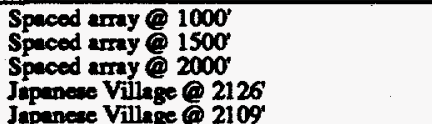 & $\begin{array}{l}0.7 \\
0.4 \\
0.1 \\
0.1 \\
0.1\end{array}$ & $\begin{array}{l}0.33 \\
0.09 \\
0.06 \\
0.05 \\
0.05\end{array}$ & $\begin{array}{l}0.31 \\
0.08 \\
0.06 \\
0.14 \\
0.12\end{array}$ & \\
\hline $\begin{array}{l}\text { P26 } \\
\text { P27 } \\
\text { P28 } \\
\text { P19 } \\
\text { P26 }\end{array}$ & 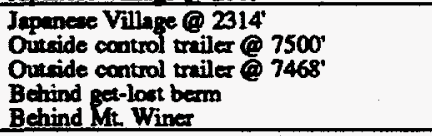 & $\begin{array}{c}0.1 \\
0.021 \\
0.021\end{array}$ & $\begin{array}{c}0.05 \\
0.015 \\
0.02\end{array}$ & $\begin{array}{l}0.12 \\
0.014 \\
0.011 \\
N \\
N S \\
\end{array}$ & \\
\hline $\begin{array}{l}\mathrm{P} 29 \\
\mathrm{P} 1 \\
\mathrm{S2} \\
\mathrm{S3} \\
\mathrm{S4} \\
\end{array}$ & $\begin{array}{l}\text { Inside control trailer @ } 7500^{\circ} \\
\text { Ceiling B4 } 300 \\
\text { Ceiling B4-300 } \\
\text { Ceiling B4-480 } \\
\text { Ceiling B4-480 } \\
\end{array}$ & $\begin{array}{c}0 \\
880 \\
880 \\
1400 \\
1400 \\
\end{array}$ & $\begin{array}{c}0.008 \\
-9 / 9 \\
-7 / 7 \\
280 \\
260 \\
\end{array}$ & $\begin{array}{l}0.006 \\
-9 / 7 \\
-7 / 6 \\
-120 / 270 \\
-110 / 260\end{array}$ & \\
\hline $\begin{array}{l}55 \\
\$ 6 \\
57\end{array}$ & $\begin{array}{l}\text { Door B4-300 } \\
\text { Door B4-300 } \\
\text { Door boh B4-300 }\end{array}$ & $\frac{125}{125}$ & $\begin{array}{l}-40 / 20 \\
-40 / 20\end{array}$ & $\begin{array}{l}-39 / 20 \\
-39 / 19 \\
\text { NS }\end{array}$ & \\
\hline Alv & $\begin{array}{l}\text { B4-480: center of floor } \\
\text { B4-480: center of floor }\end{array}$ & $\begin{array}{r}4.9 \\
4.9 \\
\end{array}$ & $\begin{array}{r}9 \\
5.7 \\
\end{array}$ & $\begin{array}{l}9.2 \\
5\end{array}$ & \\
\hline $\begin{array}{l}\text { A1T } \\
\text { A4V } \\
\text { A4R } \\
\text { A4T } \\
\text { ATN }\end{array}$ & $\begin{array}{l}\text { B4-480: center of floor } \\
\text { B4-480: SW comer of floor } \\
\text { B4-480: SW comer of floor } \\
\text { B4-480: SW comer of floor } \\
\text { B4-480: dummy camer }\end{array}$ & $\begin{array}{l}4.9 \\
4.9 \\
4.9 \\
4.9 \\
4.9 \\
\end{array}$ & $\begin{array}{c}2.4 \\
11 \\
5.4 \\
4 \\
4.7 \\
\end{array}$ & $\begin{array}{l}2.3 \\
11 \\
5.4 \\
3.6 \\
4.8 \\
\end{array}$ & \\
\hline $\begin{array}{l}\text { ATR } \\
\text { ATT } \\
\text { AZV } \\
\text { A2R } \\
\text { A2T }\end{array}$ & $\begin{array}{l}\text { B4-480. dummy camern } \\
\text { B4-480: dummy camern } \\
\text { Febetron housing: } N W \text { comer } \\
\text { Feberon housing: } N W \text { comer } \\
\text { Fobetron housing: } N W \text { comer }\end{array}$ & $\begin{array}{l}4.9 \\
4.9 \\
1.7 \\
1.7 \\
1.7\end{array}$ & $\begin{array}{l}2 \\
2 \\
>5 \\
>5 \\
>5\end{array}$ & $\begin{array}{l}2.3 \\
2 \\
>5 \\
>5 \\
>5\end{array}$ & $\begin{array}{l}\text { Signul exceeded range } \\
\text { Signal exceedded range } \\
\text { Signal exceeded range }\end{array}$ \\
\hline $\begin{array}{l}\text { A3V } \\
\text { A3R } \\
\text { A3T } \\
\text { A9R } \\
\text { A1OT }\end{array}$ & $\begin{array}{l}\text { Fobetron housing: center of floor } \\
\text { Febetron housing: center of floor } \\
\text { Febetron housing: center of floor } \\
\text { Febetron housing: inside bullthead } \\
\text { Febeting: bottle neck }\end{array}$ & $\begin{array}{l}1 \\
1 \\
1\end{array}$ & $\begin{array}{l}>5 \\
>5 \\
>5\end{array}$ & $\begin{array}{l}>5 \\
>5 \\
>5 \\
N 5 \\
N 5\end{array}$ & $\begin{array}{l}\text { Signal exceeded range } \\
\text { Signal exceeded range } \\
\text { Siggal exceeded range }\end{array}$ \\
\hline $\begin{array}{l}\text { ASV } \\
\text { ASR } \\
\text { AST } \\
\text { AGV } \\
\text { AGR } \\
\end{array}$ & $\begin{array}{l}\text { B4-300: center of laser room floor } \\
\text { B4-300: center of laser room floor } \\
\text { B4-300: center of laser room floor } \\
\text { B4-300: NW comer of laser room floor } \\
\text { B4-300: NW comer of liser noom floor }\end{array}$ & $\begin{array}{l}0.3 \\
0.3 \\
0.3 \\
0.4 \\
0.4\end{array}$ & $\begin{array}{c}0.5 \\
0.18 \\
0.076 \\
0.3 \\
0.18 \\
\end{array}$ & $\begin{array}{l}0.47 \\
0.16 \\
0.07 \\
0.3 \\
0.16 \\
\end{array}$ & \\
\hline $\begin{array}{l}\text { A6T } \\
\text { A8V } \\
\text { A8R } \\
\text { A8T } \\
\text { N1-A } \\
\end{array}$ & 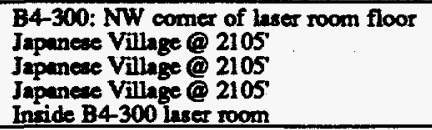 & $\begin{array}{l}0.4 \\
<0.1 \\
<0.1 \\
<0.1 \\
-120 \\
\end{array}$ & $\begin{array}{l}0.065 \\
0.19 \\
0.11 \\
0.06 \\
D E F\end{array}$ & $\begin{array}{l}0.06 \\
0.19 \\
0.11 \\
0.066 \\
D E F \\
\end{array}$ & \\
\hline $\begin{array}{l}\mathrm{N} 1-\mathrm{B} \\
\mathrm{N} 1-\mathrm{C} \text { (peak) } \\
\mathrm{N} 5-\mathrm{A} \\
\mathrm{N} 5-\mathrm{B} \\
\mathrm{N} 12 \text { (peak) }\end{array}$ & $\begin{array}{l}\text { Inside B4-300 laser room } \\
\text { Inside B4-300 laser rom } \\
\text { Inside B4-300 control room } \\
\text { Inside B4-300 control room } \\
\text { Inside B4-300 control room }\end{array}$ & -120 & $\begin{array}{l}\mathbf{N S} \\
\mathbf{N} \\
\mathbf{D E F} \\
\mathbf{N S} \\
\mathbf{N}\end{array}$ & $\begin{array}{l}\text { NS } \\
\text { NS } \\
\text { DEF } \\
\text { N } \\
\text { NS }\end{array}$ & \\
\hline $\begin{array}{l}\text { N10-A } \\
\text { N10-B } \\
\text { N11-A (peak) } \\
\text { N11-B (peak) } \\
\text { N2-A }\end{array}$ & $\begin{array}{l}\text { Inside B4-300 utility room } \\
\text { Inside B4-300 utility room } \\
\text { Inside B4-300 utility room } \\
\text { Inside B4-300 utility room } \\
\text { Entronce door B4-300 }\end{array}$ & $\begin{array}{l}-120 \\
-120 \\
\end{array}$ & $\begin{array}{l}\text { NS } \\
\text { NS } \\
>130 \\
\text { NS } \\
\text { DEF }\end{array}$ & $\begin{array}{l}\text { NS } \\
\text { NS } \\
>130 \\
\text { NS } \\
\text { DEF }\end{array}$ & \\
\hline $\begin{array}{l}\text { N2-B } \\
\text { N8-A (peak) } \\
\text { N8-B (peak) } \\
\text { N3 (peak) } \\
\text { N10-A }\end{array}$ & $\begin{array}{l}\text { Entrance door B4-300 } \\
\text { Entrance door B4-300 } \\
\text { Entrance door B4-300 } \\
\text { Outride control trailer } \\
\text { Outside control trailer }\end{array}$ & $\begin{array}{l}\sim 120 \\
<140 \\
<140\end{array}$ & $\begin{array}{l}\mathbf{N S} \\
138 \\
\mathbf{N S} \\
134 \\
122\end{array}$ & $\begin{array}{l}N S \\
138 \\
N S \\
134 \\
12\end{array}$ & \\
\hline $\begin{array}{l}\text { N10-B } \\
\text { N4-A } \\
\text { N4-B } \\
\text { N6 } \\
\text { N9 (peak) } \\
\text { N7 (peak) }\end{array}$ & $\begin{array}{l}\text { Outride control trailer } \\
\text { Inside control trailer } \\
\text { Inside control trailer } \\
\text { Inside control trailer } \\
\text { Inside control trailer } \\
\text { Crmern sution } \$ 7(9750)\end{array}$ & $\begin{array}{l}<140 \\
<140 \\
<140 \\
<140 \\
\end{array}$ & $\begin{array}{l}\text { NS } \\
\text { DEF } \\
\text { NS } \\
\text { NS } \\
135 \\
135 \\
\end{array}$ & $\begin{array}{l}S \\
\text { DEF } \\
\text { NS } \\
\text { NS } \\
135 \\
135 \\
\end{array}$ & \\
\hline
\end{tabular}


Table 5-3. Peak pressures, strains, and accelerations in POPOVER III

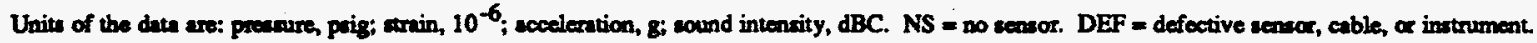

\begin{tabular}{|c|c|c|c|c|c|}
\hline Sencor & Lecation & $\begin{array}{l}\text { Predicted } \\
\text { peak } \\
\text { magnitude }\end{array}$ & $\begin{array}{l}\text { Preliminary } \\
\text { peak } \\
\text { marnitude }\end{array}$ & $\begin{array}{l}\text { Final } \\
\text { peak } \\
\text { mapnitude }\end{array}$ & Comments on final dats \\
\hline $\begin{array}{l}\mathbf{P 1} \\
\mathbf{P 2} \\
\text { P5 } \\
\text { P8 } \\
\text { P3 }\end{array}$ & $\begin{array}{l}\text { Outside B4-480 } \\
\text { Outside B4-480 } \\
\text { Outride B4-480 door } \\
\text { Outride B4-480 door } \\
\text { Inside B4-480 }\end{array}$ & $\begin{array}{l}700 \\
700 \\
12 \\
12 \\
0 \\
\end{array}$ & $\begin{array}{r}740 \\
720 \\
10 \\
12 \\
0.07 \\
\end{array}$ & $\begin{array}{l}730 \\
720 \\
13 \\
13 \\
0.07 \\
\end{array}$ & \\
\hline $\begin{array}{l}\text { P6 } \\
\text { P7 } \\
\text { P4 } \\
\text { P9 } \\
\text { P22 }\end{array}$ & $\begin{array}{l}\text { Frant outride Febetron } \\
\text { Frent outeide Febetron } \\
\text { Intide Feberon } \\
\text { Outride top B4 } 300 \\
\text { Outride tos B4300 }\end{array}$ & $\begin{array}{c}1000 \\
1000 \\
0 \\
24 \\
24 \\
\end{array}$ & $\begin{array}{l}880 \\
870 \\
0.55 \\
20.5 \\
16.3 \\
\end{array}$ & $\begin{array}{l}900 \\
870 \\
0.55 \\
20 \\
16 \\
\end{array}$ & \\
\hline $\begin{array}{l}\text { P10 } \\
\text { P13 } \\
\text { P20 } \\
\text { P14 } \\
\text { P15 }\end{array}$ & $\begin{array}{l}\text { Inside B4-300 } \\
\text { Outride door B4-300 } \\
\text { Outside doo B4-300 } \\
\text { Near N2 trailer } \\
\text { Spaced arroy } 500\end{array}$ & $\begin{array}{r}0 \\
4 \\
4 \\
-\quad 3 \\
\quad 0.9 \\
\end{array}$ & $\begin{array}{c}0.008 \\
3.8 \\
3.4 \\
3.4 \\
0.97 \\
\end{array}$ & $\begin{array}{l}0.008 \\
4 \\
3.4 \\
3.4 \\
1 \\
\end{array}$ & \\
\hline $\begin{array}{l}\text { P16 } \\
\text { P17 } \\
\text { P18 } \\
\text { P19 } \\
\text { P25 }\end{array}$ & 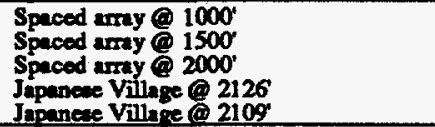 & $\begin{array}{l}0.3 \\
0.1 \\
0.1 \\
0.1 \\
0.1 \\
\end{array}$ & $\begin{array}{l}0.4 \\
0.25 \\
0.19 \\
0.22 \\
0.13\end{array}$ & $\begin{array}{l}0.4 \\
0.25 \\
0.23 \\
0.22 \\
0.13\end{array}$ & \\
\hline $\begin{array}{l}\text { P26 } \\
\text { P27 } \\
\text { P28 } \\
\text { P19 } \\
\text { P26 }\end{array}$ & $\begin{array}{l}\text { Japanese Village @ 2314' } \\
\text { Outride control triler @ 7500' } \\
\text { Outride control trailer @ 7468' } \\
\text { Behind get-lost berm } \\
\text { Behind Wt Winer }\end{array}$ & $\begin{array}{c}0.1 \\
0.021 \\
0.021\end{array}$ & $\begin{array}{l}0.14 \\
0.034 \\
0.048\end{array}$ & $\begin{array}{l}0.14 \\
0.04 \\
0.05 \\
N \\
N S \\
\end{array}$ & \\
\hline $\begin{array}{l}\mathbf{P 2 9} \\
\mathbf{S 1} \\
\mathbf{S 2} \\
\mathbf{S 3} \\
\mathbf{S 4} \\
\end{array}$ & $\begin{array}{l}\text { Inside control trailer @ } 7500 \\
\text { Ceiling B4-300 } \\
\text { Ceiling B4-300 } \\
\text { Ceiling B4-480 } \\
\text { Celing B4-480 } \\
\end{array}$ & $\begin{array}{r}0 \\
10 \\
10 \\
300 \\
300 \\
\end{array}$ & $\begin{array}{l}0.021 \\
-9 / 7.7 \\
.7 .5 / 6.3 \\
-95 / 320 \\
.90 / 290 \\
\end{array}$ & $\begin{array}{l}0.02 \\
-9 / 8 \\
-7 / 7 \\
-96 / 270 \\
-90 / 300 \\
\end{array}$ & \\
\hline $\begin{array}{l}\text { S5 } \\
56 \\
57 \\
\text { Alv } \\
\text { AlR }\end{array}$ & $\begin{array}{l}\text { Door B4-300 } \\
\text { Door B4-300 } \\
\text { Door bolt B4-300 } \\
\text { B4-480: center of floor } \\
\text { B4-480: center of floor }\end{array}$ & $\begin{array}{c}40 \\
30 \\
10 \\
5\end{array}$ & $\begin{array}{c}-46 / 23 \\
-8.7 / 52 \\
6.8 \\
5.3 \\
\end{array}$ & $\begin{array}{l}-46 / 22 \\
N 5 \\
-9 / 51 \\
9.1 \\
5.2 \\
\end{array}$ & \\
\hline $\begin{array}{l}\text { A1T } \\
\text { A4V } \\
\text { A4R } \\
\text { A4T } \\
\text { ATV }\end{array}$ & $\begin{array}{l}\text { B4-480: center of floor } \\
\text { B4-480: SW comer of floor } \\
\text { B4-480: SW comer of floor } \\
\text { B4-480: SW comer of floor } \\
\text { B4-480: dimmy cemern }\end{array}$ & $\begin{array}{c}3 \\
10 \\
5 \\
4 \\
5 \\
\end{array}$ & $\begin{array}{l}2.2 \\
9.3 \\
4.9 \\
3.6 \\
6.1 \\
\end{array}$ & $\begin{array}{l}2.2 \\
9.3 \\
5.3 \\
3.6 \\
6.3 \\
\end{array}$ & 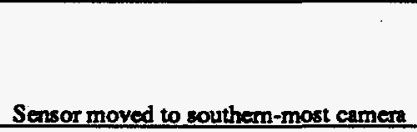 \\
\hline $\begin{array}{l}\text { ATR } \\
\text { ATT } \\
\text { A2V } \\
\text { A2R } \\
\text { A2T }\end{array}$ & $\begin{array}{l}\text { B4-480: dummy camera } \\
\text { B4-480: dummy camera } \\
\text { Fobetron housing: NW comer } \\
\text { Febetron houxing: NW camer } \\
\text { Febetron housing: NW comer }\end{array}$ & $\begin{array}{c}2 \\
2 \\
>5 \\
>5 \\
5 \\
\end{array}$ & $\begin{array}{l}3.7 \\
3 \\
>25 \\
>25 \\
>25 \\
\end{array}$ & $\begin{array}{l}3.6 \\
3.2 \\
>25 \\
>25 \\
>25\end{array}$ & $\begin{array}{l}\text { Sensor moved to southern-most camera } \\
\text { Sensor moved to southern-most camera } \\
\text { Signal exceeded range } \\
\text { Signal exceeded range } \\
\text { Signal exceeded range }\end{array}$ \\
\hline $\begin{array}{l}\text { A3V } \\
\text { A3R } \\
\text { A3T } \\
\text { A9R } \\
\text { A10T }\end{array}$ & $\begin{array}{l}\text { Febetron housing: center of floor } \\
\text { Febetron housing: center of floct } \\
\text { Febetron housing: center of floor } \\
\text { Febetron housing: inside bullkhead } \\
\text { Febetron housing: bottle reck }\end{array}$ & $\begin{array}{l}>5 \\
>5 \\
5\end{array}$ & $\begin{array}{l}>25 \\
>25 \\
13\end{array}$ & $\begin{array}{l}>25 \\
>25 \\
12 \\
N S \\
N S\end{array}$ & $\begin{array}{l}\text { Signal exceeded range } \\
\text { Signal exceeded range }\end{array}$ \\
\hline $\begin{array}{l}\text { ASV } \\
\text { ASR } \\
\text { AST } \\
\text { AGV } \\
\text { AGR }\end{array}$ & $\begin{array}{l}\text { B4-300: center of laser room floor } \\
\text { B4-300: center of laser room floor } \\
\text { B4-300: center of laser room floor } \\
\text { B4-300: NW comer of liser room floor } \\
\text { B4-300: NW comer of laser room floor }\end{array}$ & $\begin{array}{l}0.5 \\
0.2 \\
0.1 \\
0.4 \\
0.2 \\
\end{array}$ & $\begin{array}{l}0.7 \\
0.22 \\
0.11 \\
0.34 \\
0.21 \\
\end{array}$ & $\begin{array}{l}0.65 \\
0.21 \\
0.11 \\
0.32 \\
0.19 \\
\end{array}$ & · \\
\hline $\begin{array}{l}\text { A6T } \\
\text { A8V } \\
\text { A8R } \\
\text { A8T } \\
\text { N1-A }\end{array}$ & $\begin{array}{l}\text { B4-300: NW comer of laser room floor } \\
\text { Japanese Village @ 2105' } \\
\text { Japanese Village @ } 2105 \\
\text { Japanese Village } 2105 \\
\text { Inside B4-300 liser room }\end{array}$ & $\begin{array}{r}0.1 \\
0.2 \\
0.1 \\
<0.1 \\
-130 \\
\end{array}$ & 113 & $\begin{array}{l}0.11 \\
0.27 \\
0.19 \\
0.064 \\
113\end{array}$ & \\
\hline $\begin{array}{l}\text { N1-B } \\
\text { N1-C (poak) } \\
\text { N5-A } \\
\text { N5-B } \\
\text { N12 (peak) }\end{array}$ & $\begin{array}{l}\text { Inside B4-300 leser room } \\
\text { Inside B4-300 laser room } \\
\text { Inside B4-300 control room } \\
\text { Inside B4-300 control room } \\
\text { Inside B4-300 control room }\end{array}$ & $\begin{array}{r}-130 \\
-130 \\
\end{array}$ & $\begin{array}{l}\mathbf{N} \\
\mathbf{N} \\
120 \\
\mathbf{N} \\
\mathrm{DEF}\end{array}$ & $\begin{array}{l}\text { NS } \\
\text { NS } \\
120 \\
\text { NS } \\
\text { DEF } \\
\end{array}$ & \\
\hline $\begin{array}{l}\text { N10-A } \\
\text { N10-B } \\
\text { N11-A (peai) } \\
\text { N11-B (peak) } \\
\text { N2-A }\end{array}$ & $\begin{array}{l}\text { Inride B4-300 utility room } \\
\text { Inside B4-300 utility room } \\
\text { Inside B4-300 utility room } \\
\text { Inside B4-300 utility room } \\
\text { Entrance door B4-300 }\end{array}$ & $\begin{array}{r}-130 \\
-130 \\
\end{array}$ & $\begin{array}{l}\mathbf{N} \\
\mathbf{N} \\
\mathbf{D E F} \\
\mathbf{N S} \\
129\end{array}$ & $\begin{array}{l}\text { No } \\
\text { DEF } \\
\text { NS } \\
129 \\
\end{array}$ & \\
\hline $\begin{array}{l}\text { N2-B } \\
\text { N8-A (peak) } \\
\text { N8-B (peak) } \\
\text { N3 (peak) } \\
\text { N10-A }\end{array}$ & $\begin{array}{l}\text { Entrance door B4-300 } \\
\text { Entrance door B4-300 } \\
\text { Entrance door B4-300 } \\
\text { Outside control trailer } \\
\text { Outside control treiler }\end{array}$ & $\begin{array}{l}-130 \\
<140 \\
<140 \\
\end{array}$ & $\begin{array}{l}\mathbf{N} \\
140 \\
\mathbf{N S} \\
149 \\
\mathrm{DEF}\end{array}$ & $\begin{array}{l}\text { N } \\
140 \\
\text { NS } \\
149 \\
\text { DEF }\end{array}$ & - \\
\hline $\begin{array}{l}\text { N10-B } \\
\text { N4-A } \\
\text { N4-B } \\
\text { N6 } \\
\text { N9 (peak) } \\
\text { N7 (peet) }\end{array}$ & $\begin{array}{l}\text { Ortside control trailer } \\
\text { Inside control trailer } \\
\text { Inside control trailer } \\
\text { Inside contral trailer } \\
\text { Inside control trailer } \\
\text { Cemern station } \approx 7(9750)\end{array}$ & $\begin{array}{l}<140 \\
<140 \\
<140 \\
<140 \\
\end{array}$ & $\begin{array}{l}\mathbf{N} \\
142 \\
\mathbf{N S} \\
\mathbf{N S} \\
141 \\
131\end{array}$ & $\begin{array}{l}\text { NS } \\
142 \\
N S \\
\text { NS } \\
141 \\
131 \\
\end{array}$ & \\
\hline
\end{tabular}


Table 5-4. Peak pressures, strains, and accelerations in POPOVER IV

Units of the dats are: prescure, poig; atrain, $10^{-6}$; sccelerntion, $\mathrm{g}$; sound intensity, dBC. NS = po sensor. DEF $=$ defective sentor, cable, $\alpha$ instrument.

\begin{tabular}{|c|c|c|c|c|c|}
\hline Seneor & Lecation & $\begin{array}{l}\text { Predlcted } \\
\text { penk } \\
\text { magnitude }\end{array}$ & $\begin{array}{l}\text { Preliminary } \\
\text { peak } \\
\text { magnitude }\end{array}$ & $\begin{array}{l}\text { Final } \\
\text { peak } \\
\text { magnitude }\end{array}$ & Comments on final data \\
\hline $\begin{array}{l}\mathbf{P} 1 \\
\text { P2 } \\
\text { P5 } \\
\text { P8 } \\
\text { P3 }\end{array}$ & $\begin{array}{l}\text { Outride B4480 } \\
\text { Outride B4-480 } \\
\text { Outride B4-480 door } \\
\text { Outside B4-480 door } \\
\text { Invide B4-480 }\end{array}$ & $\begin{array}{c}540 \\
540 \\
85 \\
85 \\
0\end{array}$ & $\begin{array}{c}480 \\
630 \\
17 \\
19 \\
0.05\end{array}$ & $\begin{array}{l}480 \\
630 \\
17 \\
18 \\
0.06\end{array}$ & Sensor or cable darnage after peak? \\
\hline $\begin{array}{l}\mathrm{P6} \\
\mathrm{P} 7 \\
\mathrm{P4} \\
\mathrm{P9} \\
\mathrm{P} 22\end{array}$ & $\begin{array}{l}\text { Front outside Febetron } \\
\text { Front outside Febetron } \\
\text { Inside Feberon } \\
\text { Outride top B4300 } \\
\text { Outride top B4-300 }\end{array}$ & $\begin{array}{c}2350 \\
2350 \\
0 \\
54 \\
54\end{array}$ & $\begin{array}{l}0.07 \\
41 \\
47\end{array}$ & $\begin{array}{l}\mathbf{N} \\
\mathbf{N} \\
0.64 \\
41 \\
48\end{array}$ & \\
\hline $\begin{array}{l}\text { P10 } \\
\text { P13 } \\
\text { P20 } \\
\text { P14 } \\
\text { P15 }\end{array}$ & 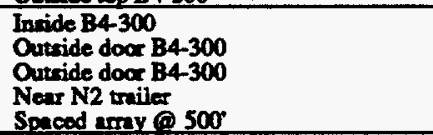 & $\begin{array}{c}0 \\
9 \\
9 \\
7.5 \\
2 \\
\end{array}$ & $\begin{array}{c}0.013 \\
6.7 \\
6.9 \\
9.4 \\
1.8 \\
\end{array}$ & $\begin{array}{l}0.014 \\
6.7 \\
6.8 \\
18 \\
1.8 \\
\end{array}$ & $\begin{array}{l}\text { Peak of reflected presoure } \\
\text { Peak of reflected presaure }\end{array}$ \\
\hline $\begin{array}{l}\text { P16 } \\
\text { P17 } \\
\text { P18 } \\
\text { P19 } \\
\text { P25 }\end{array}$ & $\begin{array}{l}\text { Spaced array } 1000^{\circ} \\
\text { Spaced array } 1500^{\circ} \\
\text { Spaced amay } 2000^{\circ} \\
\text { Japences Village } 2126 \\
\text { Jepenese Villape } 2109^{\circ}\end{array}$ & $\begin{array}{l}1 \\
0.5 \\
0.2 \\
0.2 \\
0.2 \\
\end{array}$ & $\begin{array}{l}0.65 \\
0.41 \\
0.3 \\
0.23\end{array}$ & $\begin{array}{l}0.61 \\
0.4 \\
0.28 \\
0.24 \\
0.25 \\
\end{array}$ & \\
\hline $\begin{array}{l}\text { P26 } \\
\text { P27 } \\
\text { P28 } \\
\text { P19 } \\
\text { P26 }\end{array}$ & 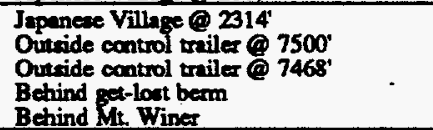 & $\begin{array}{r}0.2 \\
0.04 \\
0.04\end{array}$ & & $\begin{array}{l}0.24 \\
0.05 \\
0.06 \\
N \\
N S\end{array}$ & \\
\hline $\begin{array}{l}\text { P29 } \\
\text { S1 } \\
\text { S2 } \\
\text { S3 } \\
\text { S4 }\end{array}$ & $\begin{array}{l}\text { Inside control trailer @ 7500 } \\
\text { Ceiling B4-300 } \\
\text { Celing B4-300 } \\
\text { Celing B4-480 } \\
\text { Ceiling B4-480 }\end{array}$ & $\begin{array}{c}0 \\
34 \\
34 \\
1100 \\
1100\end{array}$ & $\begin{array}{c}-9 / 9 \\
-15 / 8 \\
540 \\
550\end{array}$ & $\begin{array}{l}\text { DEF } \\
-9 / 9 \\
-14 / 8 \\
-140 / 540 \\
-120 / 560\end{array}$ & \\
\hline $\begin{array}{l}55 \\
\$ 6\end{array}$ & $\begin{array}{l}\text { Door B4-300 } \\
\text { Door B4-300 }\end{array}$ & 300 & $-160 / 110$ & $-158 / 105$ & \\
\hline $\begin{array}{l}\text { S7 } \\
\text { Alv } \\
\text { AlR }\end{array}$ & $\begin{array}{l}\text { Door bolt B4-300 } \\
\text { B4-480: center of floor } \\
\text { B4-480: center of floor }\end{array}$ & $\begin{array}{c}300 \\
10 \\
5\end{array}$ & $\begin{array}{l}-210 \\
4.8 \\
2.8\end{array}$ & $\begin{array}{l}-215 / 37 \\
4.7 \\
2.6\end{array}$ & \\
\hline $\begin{array}{l}\text { A1T } \\
\text { A4V } \\
\text { A4R } \\
\text { A4T } \\
\text { ATV }\end{array}$ & $\begin{array}{l}\text { B4-480: center of floor } \\
\text { B4-480: SW comer of floor } \\
\text { B4-480: SW comer of fioor } \\
\text { B4-480: SW comer of floor } \\
\text { B4-480: dummy cimera }\end{array}$ & $\begin{array}{c}3 \\
10 \\
5 \\
4 \\
7 \\
\end{array}$ & $\begin{array}{r}2.1 \\
11.4 \\
5.5 \\
5.5 \\
4.7 \\
\end{array}$ & $\begin{array}{l}2.2 \\
11.4 \\
5.4 \\
5.5 \\
4.7 \\
\end{array}$ & \\
\hline $\begin{array}{l}\text { ATR } \\
\text { ATT } \\
\text { A2R } \\
\text { AZT }\end{array}$ & $\begin{array}{l}\text { B4-480: dummy camera } \\
\text { B4-480: dummy cemera } \\
\text { Febetron housing: NW camer } \\
\text { Fobetroa housing: NW camer } \\
\text { Febetron housing: NW camer }\end{array}$ & $\begin{array}{l}4 \\
3 \\
>25 \\
>25 \\
25 \\
\end{array}$ & $\begin{array}{c}3.5 \\
2.5 \\
>100 \\
60 \\
75 \\
\end{array}$ & $\begin{array}{l}3.4 \\
2.6 \\
>100 \\
57 \\
75 \\
\end{array}$ & Signal exceeded range \\
\hline $\begin{array}{l}\text { A3V } \\
\text { A3R } \\
\text { A9R } \\
\text { A10T }\end{array}$ & $\begin{array}{l}\text { Febetron housing: center of floor } \\
\text { Febetron housing: center of floor } \\
\text { Febetron housing: center of floor } \\
\text { Febetron housing: inside bullkhead } \\
\text { Febetron housing: bottle rect }\end{array}$ & $\begin{array}{l}>25 \\
>25 \\
25 \\
>25 \\
25 \\
\end{array}$ & $\begin{array}{l}60 \\
55 \\
45\end{array}$ & $\begin{array}{l}38 \\
55 \\
46 \\
\text { DEF } \\
\text { DEF }\end{array}$ & \\
\hline $\begin{array}{l}\text { ASV } \\
\text { ASR } \\
\text { AST } \\
\text { AGV } \\
\text { AGR }\end{array}$ & $\begin{array}{l}\text { B4-300: cenver of laser room floor } \\
\text { B4-300: center of laser rom floor } \\
\text { B4-300: center of laser room floor } \\
\text { B4-300: NW comer of laser room floor } \\
\text { B4-300: NW comer of leser room floor }\end{array}$ & $\begin{array}{l}0.7 \\
0.7 \\
0.7 \\
0.7 \\
0.7\end{array}$ & $\begin{array}{c}0.7 \\
0.45 \\
0.25 \\
0.6 \\
0.45 \\
\end{array}$ & $\begin{array}{l}0.65 \\
0.43 \\
0.23 \\
0.54 \\
0.41\end{array}$ & \\
\hline $\begin{array}{l}\text { A6T } \\
\text { A8V } \\
\text { A8R } \\
\text { A8T } \\
\text { N1-A }\end{array}$ & 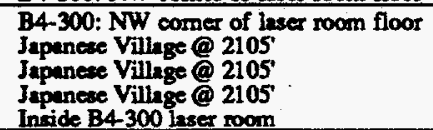 & $\begin{array}{c}0.7 \\
0.1 \\
0.1 \\
0.1 \\
-130\end{array}$ & $\begin{array}{l}0.16 \\
>0.2 \\
127\end{array}$ & $\begin{array}{l}0.16 \\
0.26 \\
0.2 \\
0.12 \\
127\end{array}$ & \\
\hline $\begin{array}{l}\text { N1-B } \\
\text { N1-C (peak) }\end{array}$ & $\begin{array}{l}\text { Incide B4300 laser room } \\
\text { Inside B4-300 laser room }\end{array}$ & -130 & $\begin{array}{l}128 \\
\text { NS }\end{array}$ & 128 & \\
\hline $\begin{array}{l}\text { N5-A } \\
\text { N5-B } \\
\text { N12 (peak) }\end{array}$ & $\begin{array}{l}\text { Inside B4-300 control noom } \\
\text { Inside B4-300 control room } \\
\text { Inside B4-300 control room }\end{array}$ & $\begin{array}{l}-130 \\
-130 \\
-130\end{array}$ & $\begin{array}{l}121 \\
123 \\
113\end{array}$ & $\begin{array}{l}121 \\
123 \\
113\end{array}$ & \\
\hline $\begin{array}{l}\text { N10-A } \\
\text { N1Q-B } \\
\text { N11-A (peak) } \\
\text { N11-B (peak) } \\
\text { N2-A }\end{array}$ & $\begin{array}{l}\text { Inside B4-300 wility room } \\
\text { Inside B4-300 urility room } \\
\text { Inside B4-300 utility room } \\
\text { Inside B4-300 utility room } \\
\text { Entrence door B4-300 }\end{array}$ & $\begin{array}{l}-130 \\
-130\end{array}$ & $\begin{array}{c}\mathbf{N S} \\
\mathbf{N} \\
\mathbf{1 3 0} \\
\mathrm{NS} \\
141\end{array}$ & $\begin{array}{l}\text { NS } \\
N S \\
>130 \\
N S \\
141\end{array}$ & Signal exceeded range \\
\hline $\begin{array}{l}\text { N2-B } \\
\text { N8-A (peak) } \\
\text { N8-B (penk) }\end{array}$ & $\begin{array}{l}\text { Entrance door B4-300 } \\
\text { Entrance door B4-300 } \\
\text { Entrance door B4-300 }\end{array}$ & $\begin{array}{l}-130 \\
-130\end{array}$ & $\begin{array}{l}141 \\
140 \\
\text { NS }\end{array}$ & $\begin{array}{l}141 \\
140 \\
\text { NS }\end{array}$ & \\
\hline $\begin{array}{l}\text { N3 (peak) } \\
\text { N10-A }\end{array}$ & $\begin{array}{l}\text { Outside control trailer } \\
\text { Outside control trailer }\end{array}$ & $\begin{array}{l}>140 \\
>140\end{array}$ & $\begin{array}{l}136 \\
146\end{array}$ & $\begin{array}{l}136 \\
146\end{array}$ & \\
\hline $\begin{array}{l}\text { N10-B } \\
\text { N44A } \\
\text { N4-B } \\
\text { N6 } \\
\text { N9 (peak) } \\
\text { N7 (peak) }\end{array}$ & $\begin{array}{l}\text { Outside control trailer } \\
\text { Inside control trailer } \\
\text { Inside control trailer } \\
\text { Inside control trailer } \\
\text { Inside control trailer } \\
\text { Cemera station } \# \text { ? } 99750 \text { ) }\end{array}$ & $\begin{array}{l}>140 \\
>140 \\
>140 \\
>140 \\
>140 \\
>140\end{array}$ & $\begin{array}{l}144 \\
134 \\
135 \\
\text { NS } \\
141 \\
139 \\
\end{array}$ & $\begin{array}{l}144 \\
134 \\
135 \\
N S \\
141 \\
139\end{array}$ & \\
\hline
\end{tabular}


Table 5-5. Peak pressures, strains, and accelerations in POPOVER V

Units of the data are: proseure, priz; strin, $10^{-6}$; acceleration, g; sound intensity, dBC. NS = no sensor. DEF = defective sensor, cable, or instrument.

\begin{tabular}{|c|c|c|c|c|c|}
\hline Sensor & Location & $\begin{array}{l}\text { Predicted } \\
\text { peak } \\
\text { merniltude }\end{array}$ & $\begin{array}{l}\text { Preliminary } \\
\text { peak } \\
\text { mepnltude }\end{array}$ & $\begin{array}{l}\text { Final } \\
\text { peak } \\
\text { mernitude }\end{array}$ & Comments on final dete \\
\hline $\begin{array}{l}\mathrm{P} 1 \\
\mathrm{P} 2 \\
\mathrm{P} 5 \\
\mathrm{~PB} \\
\mathrm{P3}\end{array}$ & $\begin{array}{l}\text { Outside B4-480 } \\
\text { Outside B4-480 } \\
\text { Outside B4-480 door } \\
\text { Outride B4480 door } \\
\text { Invide B4-480 }\end{array}$ & $\begin{array}{c}770 \\
770 \\
20 \\
20 \\
0\end{array}$ & $\begin{array}{c}540 \\
530 \\
19 \\
21 \\
0.014\end{array}$ & $\begin{array}{l}620 \\
530 \\
19 \\
20 \\
0.012\end{array}$ & Series of reflectod waves \\
\hline $\begin{array}{l}\mathrm{PF} \\
\mathrm{P} 7 \\
\mathrm{P4} \\
\mathrm{P9} \\
\mathrm{P} 22\end{array}$ & $\begin{array}{l}\text { Front oustide Febetron } \\
\text { Front outside Fobetron } \\
\text { Inside Feberon } \\
\text { Outside top B4-300 } \\
\text { Outside top B4-300 }\end{array}$ & $\begin{array}{c}0 \\
50 \\
50\end{array}$ & $\begin{array}{l}0.42 \\
45 \\
45\end{array}$ & $\begin{array}{l}5 \\
N 5 \\
0.43 \\
45 \\
45 \\
\end{array}$ & \\
\hline $\begin{array}{l}\text { P10 } \\
\text { P13 } \\
\text { P20 } \\
\text { P14 } \\
\text { P15 }\end{array}$ & 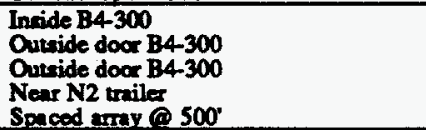 & $\begin{array}{l}0 \\
9 \\
9 \\
20 \\
2.5\end{array}$ & $\begin{array}{c}0.014 \\
8.7 \\
9.2 \\
9 \\
1.1 \\
\end{array}$ & $\begin{array}{l}0.013 \\
88.6 \\
8.9 \\
9 \\
1.1 \\
\end{array}$ & $\begin{array}{l}\text { Reflected peak with spurious peaks } \\
\text { Reflected peak }\end{array}$ \\
\hline $\begin{array}{l}\text { P16 } \\
\text { P17 } \\
\text { P18 } \\
\text { P19 } \\
\text { P25 }\end{array}$ & 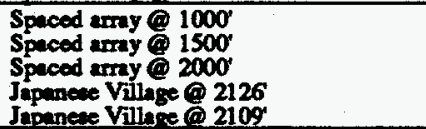 & $\begin{array}{l}1 \\
0.6 \\
0.4 \\
0.25\end{array}$ & $\begin{array}{l}0.88 \\
0.64 \\
0.43\end{array}$ & $\begin{array}{l}0.68 \\
0.45 \\
0.29 \\
N 5 \\
0.35\end{array}$ & \\
\hline $\begin{array}{l}\text { P26 } \\
\text { P27 } \\
\text { P28 } \\
\text { P19 } \\
\text { P26 }\end{array}$ & 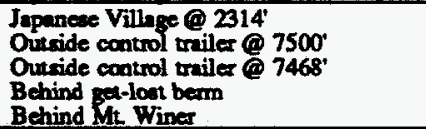 & $\begin{array}{l}0.25 \\
0.05 \\
0.05 \\
20\end{array}$ & 4.5 & $\begin{array}{l}\mathbf{N} \\
0.044 \\
0.037 \\
4.4 \\
0.35\end{array}$ & \\
\hline $\begin{array}{l}\mathrm{P} 29 \\
\mathrm{~S} 1 \\
\mathrm{S2} \\
\mathrm{S3} \\
\mathrm{S4}\end{array}$ & $\begin{array}{l}\text { Intide control trailer @ } 7500 \\
\text { Ceiling B4-300 } \\
\text { Ceiling B4300 } \\
\text { Ceiling B4-480 } \\
\text { Ceiling B4-480 }\end{array}$ & $\begin{array}{r}0 \\
20 \\
20 \\
800 \\
800\end{array}$ & $\begin{array}{l}-12 \\
-12 \\
179 \\
174\end{array}$ & $\begin{array}{l}0.019 \\
-12 / 9 \\
-12 / 9 \\
-150 / 180 \\
-150 / 175\end{array}$ & \\
\hline $\begin{array}{l}55 \\
\$ 6\end{array}$ & $\begin{array}{l}\text { Door B4-300 } \\
\text { Door B4-300 }\end{array}$ & 200 & 209 & $\begin{array}{l}-176 / 210 \\
N S\end{array}$ & \\
\hline $\begin{array}{l}\text { s7 } \\
\text { A1v } \\
\text { AlR }\end{array}$ & $\begin{array}{l}\text { Door bolt B4-300 } \\
\text { B4-480: center of floor } \\
\text { B4-480: center of floor }\end{array}$ & $\begin{array}{c}300 \\
10 \\
6 \\
\end{array}$ & $\begin{array}{r}-195 \\
6.9 \\
7.4 \\
\end{array}$ & $\begin{array}{l}-192 / 81 \\
6.8 \\
7.3\end{array}$ & \\
\hline $\begin{array}{l}\text { A1T } \\
\text { A4V } \\
\text { A4R } \\
\text { A4T } \\
\text { ATV }\end{array}$ & $\begin{array}{l}\text { B4-480: center of floor } \\
\text { B4-480: SW comer of floor } \\
\text { B4-480: SW comer of floor } \\
\text { B4-480: SW comer of floor } \\
\text { B4-480: dummy camer }\end{array}$ & $\begin{array}{c}3 \\
11 \\
6 \\
5 \\
6\end{array}$ & $\begin{array}{l}3 \\
11 \\
6.8 \\
4.8 \\
3.9\end{array}$ & $\begin{array}{l}2.9 \\
11 \\
6.8 \\
4.8 \\
3.8\end{array}$ & \\
\hline $\begin{array}{l}\text { ATR } \\
\text { ATT } \\
\text { A2V } \\
\text { A2R } \\
\text { A2T }\end{array}$ & $\begin{array}{l}\text { B4-480: dummy cumera } \\
\text { B4-480: dummy camera } \\
\text { Feberon housing: NW cocoer } \\
\text { Fobetron housing: NW comer } \\
\text { Febetron heasing: NW comer }\end{array}$ & $\begin{array}{c}4 \\
3 \\
200 \\
100 \\
50\end{array}$ & $\begin{array}{l}5 \\
2.3 \\
300 \\
139 \\
165\end{array}$ & $\begin{array}{l}5 \\
2.3 \\
>300 \\
140 \\
180 \\
\end{array}$ & Signal exceeded 300 g system mange \\
\hline $\begin{array}{l}\text { A3V } \\
\text { A3R } \\
\text { A3T } \\
\text { A9R } \\
\text { AlOT }\end{array}$ & $\begin{array}{l}\text { Febetron housing: center of floor } \\
\text { Febetron housing: center of floor } \\
\text { Febetron housing: center of floor } \\
\text { Foberon bousing: inside bulkhead } \\
\text { Febetron housing: bottle nck }\end{array}$ & $\begin{array}{c}50 \\
60 \\
50 \\
200 \\
50 \\
\end{array}$ & $\begin{array}{l}146 \\
204 \\
300\end{array}$ & $\begin{array}{l}210 \\
210 \\
>300 \\
\text { DEF } \\
\text { DEF }\end{array}$ & $\begin{array}{l}\text { Signal exceeded } 100 \text { g sensor range } \\
\text { Signal exceeded } 100 \mathrm{~g} \text { sensor range } \\
\text { Signal exceeded } 100 \mathrm{~g} \text { sensor range }\end{array}$ \\
\hline $\begin{array}{l}\text { ASV } \\
\text { ASR } \\
\text { AST } \\
\text { AGR }\end{array}$ & $\begin{array}{l}\text { B4-300: center of laser room floor } \\
\text { B4-300: center of laser rome floor } \\
\text { B4-300: center of herer room floox } \\
\text { B4-300: NW comer of laser room floor } \\
\text { B4-300: NW comer of laser room floor }\end{array}$ & $\begin{array}{l}0.8 \\
0.5 \\
0.3 \\
0.8 \\
0.5\end{array}$ & $\begin{array}{l}0.9 \\
0.7 \\
0.33 \\
0.65 \\
0.68\end{array}$ & $\begin{array}{l}0.85 \\
0.67 \\
0.31 \\
0.6 \\
0.62\end{array}$ & \\
\hline $\begin{array}{l}\text { A6T } \\
\text { A8V } \\
\text { A8R } \\
\text { A8T } \\
\text { N1-A }\end{array}$ & $\begin{array}{l}\text { B4-300: NW comer of laser room floor } \\
\text { Japanese Village @ 2105' } \\
\text { Japanese Village } 2105^{\prime} \\
\text { Japanese Village } 2105^{\prime} \\
\text { Intide B4-300 later room }\end{array}$ & $\begin{array}{c}0.3 \\
0.3 \\
0.2 \\
0.1 \\
-130\end{array}$ & 0.31 & $\begin{array}{l}0.3 \\
0.24 \\
0.12 \\
0.78 \\
130\end{array}$ & \\
\hline $\begin{array}{l}\text { N1-B } \\
\text { N1-C (peak) } \\
\text { N5-A } \\
\text { NS-B } \\
\text { N12 (peak) }\end{array}$ & $\begin{array}{l}\text { Inxide B4-300 leser room } \\
\text { Inride B4-300 leser room } \\
\text { Inside B4-300 control room } \\
\text { Inside B4-300 control rom } \\
\text { Inxide B4-300 control room }\end{array}$ & $\begin{array}{l}-130 \\
-130 \\
-130 \\
\sim 130 \\
-130 \\
\sim 130\end{array}$ & $\begin{array}{l}130 \\
132 \\
123 \\
123 \\
131\end{array}$ & $\begin{array}{l}130 \\
132 \\
123 \\
123 \\
131\end{array}$ & \\
\hline $\begin{array}{l}\text { N10-A } \\
\text { N10-B } \\
\text { N11-A (peak) } \\
\text { N11-B (peak) } \\
\text { N2-A }\end{array}$ & $\begin{array}{l}\text { Inside B4-300 utility room } \\
\text { Inside B4-300 utility room } \\
\text { Inside B4-300 utility room } \\
\text { Inside B4-300 utility room } \\
\text { Entrance door B4-300 }\end{array}$ & $\begin{array}{l}-130 \\
-130 \\
-130 \\
-130 \\
-130 \\
-140\end{array}$ & $\begin{array}{c}129 \\
126 \\
>130 \\
134 \\
141 \\
\end{array}$ & $\begin{array}{l}129 \\
126 \\
>130 \\
134 \\
141 \\
\end{array}$ & $\begin{array}{l}\text { New location for } N 10-A \\
\text { New location for } N 10-B \\
\text { Exceeded } 130 \mathrm{dBA} \text { range of instrument }\end{array}$ \\
\hline $\begin{array}{l}\text { N2-B } \\
\text { N8-A (peak) } \\
\text { N8-B (peak) } \\
\text { N3 (peak) } \\
\text { N10-A }\end{array}$ & $\begin{array}{l}\text { Entrance door B4-300 } \\
\text { Entrance door B4-300 } \\
\text { Entrance door B4-300 } \\
\text { Outside control trailer } \\
\text { Outside control treiler }\end{array}$ & $\begin{array}{l}-140 \\
-140 \\
-140 \\
>140\end{array}$ & $\begin{array}{l}141 \\
141 \\
141 \\
135 \\
\text { NS } \\
\end{array}$ & $\begin{array}{l}141 \\
141 \\
141 \\
135 \\
\mathrm{NS} \\
\end{array}$ & \\
\hline $\begin{array}{l}\text { N10-B } \\
\text { N44A } \\
\text { N4-B } \\
\text { N6 } \\
\text { N9 (peak) } \\
\text { N7 (peak) }\end{array}$ & $\begin{array}{l}\text { Outside control trailer } \\
\text { Inside control trailer } \\
\text { Inside control trailer } \\
\text { Inside control trailer } \\
\text { Inside control trailer } \\
\text { Camere station } \# 7 \text { (9750) }\end{array}$ & $\begin{array}{l}>140 \\
>140 \\
>140 \\
>140\end{array}$ & $\begin{array}{l}N \\
132 \\
132 \\
N S \\
141 \\
141 \\
\end{array}$ & $\begin{array}{l}S \\
132 \\
132 \\
N S \\
141 \\
141\end{array}$ & \\
\hline
\end{tabular}




\section{Section 6}

\section{Analysis of the POPOVER Test Series}

\subsection{Pressure}

A number of pressure measurements were made to certify yield, confirm calculated pressures, validate the structural calculations that were used to certify the structural integrity of the two bunkers and the Febetron housing, and verify that the pressure exposure to personnel was safe. The type of pressure sensors used and their placement are shown in Section 5.

\section{POPOVER I}

The first POPOVER test, POPOVER I, was a 512-lb TNT charge consisting of two spherical 256-lb center-detonated charges. The charges were placed $15 \mathrm{ft}(4.5 \mathrm{~m})$ from the face of Bunker B4-480. The placement of the two simultaneously detonated spheres is suspected to have caused a radial focusing effect on the close-in pressure gauges located at the B4-480 center optics port. These pressure readings ( $\mathrm{P} 1>960 \mathrm{psig}$ and $\mathrm{P} 2=520 \mathrm{psig}$ ) were higher than the 440 psig predicted.

The pressures measured outside the door to the camera facility (B4-480) were 7.7 to 8.1 psig vs less than 44 psig predicted. Because the camera room door is located at the end of a subsurface tunnel, the difference is attributed to the effect of the turns the pressure pulse must take to reach the door and to the shielding effect of the tunnel.

The pressure outside Bunker B4-300, on top of the overburden, was within 18\% of the predicted value of $15 \mathrm{psig}$. Outside the B4-300 door, the reflected pressures were less than the predicted values.

The measured pressures near the tube-trailer locations, along the spaced array, at the nearby ruins of the mock Japanese Village, and at the control trailer were all less than the predicted values.

\section{POPOVER II}

POPOVER II consisted of a spherical 1,000-lb center-detonated TNT charge located $15 \mathrm{ft}$ $(4.5 \mathrm{~m})$ from the face of Bunker B4-480. Measured pressures at the center optics port were 770 and $680 \mathrm{psig}$. The predicted value, $640 \mathrm{psig}$, was calculated assuming a $15-\mathrm{ft}(4.5-\mathrm{m})$ distance to the charge, but the center-optics-port pressure transducers were actually 16 in. $(0.4 \mathrm{~m})$ closer - namely, at $13 \mathrm{ft} .8 \mathrm{in}$. $(4.1 \mathrm{~m})$. This difference explains why the measured values were slightly larger than predicted. 
The pressures measured outside the door to the camera facility (B4-480) were 12 psig vs 44 psig predicted. The difference is once again attributed to the effect of the turns required for the pressure pulse to reach the door and to the shielding effect of the tunnel.

Pressures measured on top of B4-300 were 25 and 17 psig vs 24.5 psig predicted. Outside the B4-300 door the reflected pressure was measured at $4.6 \mathrm{psig}$ vs $3.8 \mathrm{psig}$ predicted.

A pressure higher than predicted was observed on a transducer located on the front face of the Febetron house. Indications are that this sensor was struck by a flying object. The adjacent sensor did not show signs of being impacted by flying objects; it recorded greater than 900 psig vs 454 psig predicted.

The measured pressures near the tube-trailer locations, along the spaced array, at the Japanese Village, and at the control trailer were all within $0.04 \mathrm{psig}$ of the predicted values.

\section{POPOVER III}

POPOVER III consisted of a spherical 1,000-lb center-detonated TNT charge located $15 \mathrm{ft}$ $(4.5 \mathrm{~m})$ from the face of Bunker B4-480. Measured pressures at the center optics port were 730 and $720 \mathrm{psig}$; the predicted value was 700 psig.

The pressure measured outside the door to the camera facility (B4-480) was 14 psig vs 12 psig predicted. There was a slight measurable pressure inside B4-480. The leakage was traced to a communication duct that had been left open. Administrative procedures have been put in place to eliminate this situation. These low-level sound-type pressures were well within acceptable limits.

The pressure measured on top of B4-300 was 20 psig vs 24 psig predicted. Outside the B4-300 door the reflected pressure was equal to the 4.0 psig predicted pressure.

The pressures measured on the front face of the Febetron house were 900 and 870 psig vs 1,000 psig predicted. There was also a slight measurable pressure $(0.55 \mathrm{psig})$ inside the Febetron house. This low-level sound-type pressure was well within acceptable limits.

The pressure near the tube-trailer location was measured at 3.4 psig vs 3.0 psig predicted.

The measured pressures along the spaced array were slightly higher (by $0.15 \mathrm{psig}$ ) than the predicted values. Pressures to $0.05 \mathrm{psig}$ were measured at the control trailer $(0.021 \mathrm{psig}$ predicted).

\section{POPOVER IV}

POPOVER IV consisted of three spherical 1,000-lb side-detonated TNT charges and one top-detonated 2,000-lb spherical charge located $27 \mathrm{ft}(8.2 \mathrm{~m})$ from the face of B4-480. All charges were detonated simultaneously, each with $5 \mathrm{lb}$ of $\mathrm{C} 4$. Measured pressures at the center optics port were 480 and 630 psig vs 540 psig predicted.

The pressures measured outside the door to the camera facility (B4-480) were 17 and $18 \mathrm{psig}$ vs 85 psig predicted. There was a slight measurable pressure $(0.06 \mathrm{psig})$ inside the bunker. 
The pressures at the front face of the Febetron house could no longer be measured because a gravel berm had been placed between the explosive charges and the Febetron house. There was a slight measurable pressure ( $0.064 \mathrm{psig})$ inside the Febetron house.

Pressures measured on top of B4-300 were 41 and 48 psig vs 54 psig predicted. Outside the B4-300 door the reflected pressures were measured at 6.7 and 6.8 psig vs 9 psig predicted.

The pressure near the tube-trailer location was measured at 18 psig vs 7.5 psig predicted. (The measured value was the peak of the reflected pulse, whereas the prediction did not include reflections.)

The pressures at the spaced array, the Japanese Village, and the control trailers were within $0.39 \mathrm{psig}$ of the predicted values.

\section{POPOVER V}

POPOVER V consisted of a 1,256-lb TNT charge comprised of one spherical 1,000-lb center-detonated TNT charge and one spherical 256-lb center-detonated TNT charge located $15 \mathrm{ft}(4.5 \mathrm{~m})$ from the face of B4-480 and a 6,534-lb TNT charge located $27 \mathrm{ft}(8.2 \mathrm{~m})$ from the face of B4-480. The 6,534-lb charge consisted of two spherical 2,000-1b TNT charges, two spherical 1,000-lb TNT charges, and two spherical center-detonated 256-lb TNT charges. The 1,000- and 2,000-lb charges were detonated by 5-lb C4 charges mounted on the outer surface of the spheres ( $20 \mathrm{lb}$ of C4 is equivalent to $22.5 \mathrm{lb}$ of TNT). The total amount of HE was equivalent to 7,790 lb of TNT; all charges were detonated simultaneously.

This test was considered an interference shot - that is, a shot in which one fireball interferes with another. Because both charges were detonated at the same time, the close-in measured pressures, and in fact all close-in blast-related quantities, did not match predicted values. For example, the pressures measured at the B4-480 center optics port were 620 and $530 \mathrm{psig}$, whereas the predicted value was $770 \mathrm{psig}$. The interference had very little effect on the long-range pressure measurements, which were within $20 \%$ of the predicted values. For example, the reflected pressures at the B4-480 door were very close to the predicted value ( 19 and 20 psig vs $20 \mathrm{psig}$ ).

Similarly, the pressure on top of the berm above B4-300, where the shot fireballs did not interfere, was measured at 45 psig vs 50 psig predicted. The reflected pressure at the B4-300 door was measured at 8.6 and 8.9 psig vs 9 psig predicted.

Near the tube-trailer location, 9 psig was measured vs 20 psig predicted. This difference is attributed to the fact that the sensor was relocated because a nitrogen tube trailer had been placed where a similar trailer will be located in all future BEEF tests. This 9-psig pressure pulse caused buckling of the thin metal panel double doors at the rear of the trailer that protect the trailer's instrumentation. In addition, two plastic heavy-oil seals on the left side (the more open side) of the trailer were dislodged from the end of the axles. It is believed that the negative pressure pulse extracted the seals from their normal position.

The pressures measured along the spaced array were all very close to those predicted. 
For facilities that will experience explosive-generated pressures, LLNL has adopted a policy of subjecting the facilities to pressures that are $25 \%$ higher than those planned. POPOVER V was this overtest; it established conservative operational limits of $1,000 \mathrm{lb}$ of TNT at $15 \mathrm{ft}(4.5 \mathrm{~m})$ and $5,000 \mathrm{lb}$ of TNT at $27 \mathrm{ft}(8.2 \mathrm{~m})$.

\section{Summary and Conclusions}

In general, pressure measurements from the POPOVER series were within 20\% of the values predicted. This $20 \%$ is consistent with historical results and has to do with variations in detonator placement, atmospheric conditions, wind speed, type and formulation of $\mathrm{HE}$, and sensor problems. The pressure measurements confirm that full blast yield was achieved, which implies that the BEEF structures were subjected to the expected dynamic pressure loadings.

Section 4 discusses the pressure limits established by Ref. 4 . For a risk factor of $\mathrm{K}=24 \mathrm{ft} / 1 \mathrm{~b}^{1 / 3}$ the side-on pressure limit, Pso, is $2.3 \mathrm{psig}(16 \mathrm{kPa})$; for $\mathrm{K}=328 \mathrm{ft} / 1 \mathrm{~b}^{1 / 3}$ the limit is $0.055 \mathrm{psig}(380 \mathrm{~Pa})$. This lower limit - the OSHA-established sound-pressure limit of $140 \mathrm{~dB}$ - is not exceeded for the personnel-occupied areas in Bunker B4-300 with the door closed.

For future tests it is recommended that the thin metal double doors on the tube trailer be left open or removed and some arrangement made to keep the heavy-oil axle seals in place. In addition, some type of terminal-velocity fragment protection should be provided for the nitrogen tube trailer to eliminate the possibility that a fragment missile will cause a large release of nitrogen.

The maximum POPOVER HE charge weight (with its associated $R / W^{1 / 3}$ ) will be used to produce an HE placement map for future testing at BEEF. This map will show, for various amounts of HE charge weights, isobars of explosive mass that represent the safe minimum placement distance for future tests. Even though the POPOVER certification tests were for a specific maximum amount $(5,000$ and $1,000 \mathrm{lb}$ of TNT at 27 and $15 \mathrm{ft}$, respectively, from the face of B4-480), future BEEF tests may include larger charges with proper placement of the HE charges - i.e., at greater distances and with adherence to standard blast-effects engineering practice. The use of this map to establish proper distances should be augmented to include assessment of the potential damage from shrapnel and assessment of possibly unacceptable noise levels (i.e., greater than $140 \mathrm{~dB}$ ) in the control room (B4-300) and at the far-field explosion-zone limits. It is recommended that the appropriate procedures be incorporated into the standard facility operation procedures to permit larger charges as the need arises. 


\subsection{Acceleration}

\section{POPOVER I}

Acceleration measurements were taken by triaxial accelerometers at eight locations:

\begin{tabular}{|c|c|c|}
\hline Location & Placement & Accelerometer \\
\hline B4-480 & $\begin{array}{l}\text { Middle of floor } \\
\text { SW cormer } \\
\text { Dummy camera }\end{array}$ & $\begin{array}{l}\text { A1 } \\
\text { A4 } \\
\text { A7 }\end{array}$ \\
\hline Febetron house & $\begin{array}{l}\text { NW corner } \\
\text { Middle of floor }\end{array}$ & $\begin{array}{l}\mathrm{A} 2 \\
\mathrm{~A} 3\end{array}$ \\
\hline B4-300 & $\begin{array}{l}\text { Middle of laser room } \\
\text { NW corner }\end{array}$ & $\begin{array}{l}\text { A5 } \\
\text { A6 }\end{array}$ \\
\hline Japanese Village & $2105 \mathrm{ft}(642 \mathrm{~m})$ & A8 \\
\hline
\end{tabular}

The purpose of the acceleration measurements was to ensure personnel safety, primarily from falling or overturning objects and secondarily from direct effects, and to ensure the integrity of the structures and support facilities. The direct effects on structures were measured by strain gauges and visual inspection. However, the effects on equipment not present during the POPOVER test series can be evaluated based on the accelerations at the points where they will be mounted.

The results from.POPOVER I, a 512-lb TNT test, are tabulated in Section 5. The notable features are (1) early-time electronic noise obscured signals for A1, A4, and A7; (2) the Febetron house was not emplaced for this shot; and (3) accelerations in B4-300 ranged from 0.05 to $0.41 \mathrm{~g}$. Because POPOVER I was designed to be a calibration shot, the results are not too significant. A noise interference problem was identified in the instrumentation that was thought to be from a grounding problem. For POPOVER II the grounding was reconfigured.

\section{POPOVER II}

For the most part, the noise interference problem of POPOVER I was fixed in POPOVER II. The horizontal accelerations inside B4-480 ranged from 2 to $5.4 \mathrm{~g}$ (close to the predicted $4.9 \mathrm{~g}$ ), and the vertical accelerations ranged from 4.8 to $11 \mathrm{~g}$ (vs $4.9 \mathrm{~g}$ predicted). Inside B4-300 the horizontal accelerations ranged from 0.06 to $0.16 \mathrm{~g}$ (vs $0.3-0.4 \mathrm{~g}$ predicted), and the vertical accelerations ranged from 0.3 to $0.47 \mathrm{~g}$ (vs $0.3-0.4 \mathrm{~g}$ predicted). The accelerations in the Febetron house were off-scale, exceeding $5 \mathrm{~g}$. As a result the accelerometer range was increased to $25 \mathrm{~g}$ for the next test. At the POPOVER II post-mortem meeting the issue was raised as to how to determine if the accelerations are a threat to the facility or personnel. 


\section{POPOVER III}

The horizontal accelerations inside B4-480 ranged from 2.2 to $5.3 \mathrm{~g}$, and the vertical accelerations ranged from $6.3 \mathrm{~g}$ to $9.3 \mathrm{~g}$. Inside $\mathrm{B} 4-300$ the horizontal accelerations ranged from 0.11 to $0.21 \mathrm{~g}$, and the vertical accelerations ranged from 0.32 to $0.65 \mathrm{~g}$. Accelerations in the Febetron house in the vertical and radial directions were off scale, exceeding $25 \mathrm{~g}$. One of the tangential accelerations was recorded at $12 \mathrm{~g}$. At the postmortem meeting it was suggested that the high accelerations could be potentially damaging to the Febetrons, and an engineering analysis was recommended to evaluate this possibility. It was also suggested that a velocity transducer be installed to help capture motions at frequencies important to structural response. For POPOVER IV, accelerometers with a higher range (up to $100 \mathrm{~g}$ ) were installed in the Febetron house. In addition, a berm was placed in front of the Febetron house to help mitigate the effects of the pressure pulse.

The acceleration records from the Febetron house were at a very high frequency and were likely due to acoustic waves reverberating along the length of the cylindrical Febetron house. It was observed that an aerosol can and a spray bottle on the floor remained upright during the test, but the lead shutter on the inside front plate was bent. It is not inconsistent that the objects did not fall over due to high frequency accelerations, because a small amount of sliding would essentially isolate the objects. However, the accelerations could damage brittle components rigidly attached to the floor.

The duration of the positive pressure pulse at the front face of the Febetron house was about $5 \mathrm{~ms}$, which is believed to be the primary driving force causing the horizontal accelerations, at least radially. It is likely that direct ground transmission contributed somewhat to the horizontal accelerations and greatly to the vertical accelerations. It was difficult to discern the structural frequency of the Febetron house from the records. Presumably, one could integrate the record over sufficient time to get the velocities and displacements, which might better show the structural response. However, since the structural accelerations were probably quite low compared with the high-frequency values, there may have been a signal-to-noise-ratio problem. In any event, the effects of highfrequency accelerations could be greatly mitigated if the Febetron structure were mounted with some form of base isolation system that would filter out most of the input. Electronically filtering the data with a $50-\mathrm{Hz}$ low-pass filter resulted in peaks less than $6 \mathrm{~g}$.

\section{POPOVER IV}

The horizontal accelerations inside B4-480 ranged from 2.2 to $5.5 \mathrm{~g}$, and the vertical accelerations ranged from 4.7 to $11.4 \mathrm{~g}$. Inside B4-300 the horizontal accelerations ranged from 0.16 to $0.43 \mathrm{~g}$, and the vertical accelerations ranged from $0.54 \mathrm{~g}$ to $0.65 \mathrm{~g}$. The accelerations in the Febetron house in the horizontal directions ranged from 46 to $75 \mathrm{~g}$ and in the vertical direction from 38 to more than $100 \mathrm{~g}$. Again, the accelerations were at very high frequency. At the post-mortem meeting an action item was identified to integrate acceleration data from accelerometers A5 and A6 (in B4-300) to get velocities and displacements and perform a literature review to determine if there is any guidance on human tolerance limits to these motions. 
An engineering evaluation of the Febetron was completed that indicated that the machine is very robust. It was reported that the glass tube is surrounded by oil and thus can survive high accelerations. The integrated records for the Febetron house showed nonzero residual velocities. These velocities can be brought back to zero by truncating the 8- to 36-ms portion of the record before integrating. This is a questionable practice, however, because the duration of the positive pressure pulse from POPOVER III was only $5 \mathrm{~ms}$. The pressure pulse from POPOVER IV was not measured at the Febetron housing because the berm was placed in front of it. If the pressure pulse from POPOVER IV is assumed to be of the same duration, truncating the record after $8 \mathrm{~ms}$ deletes the portion of the record over which the pressure-wave part of the forcing function acts. Nevertheless, the Febetron house was observed to move approximately $1 \mathrm{in}$. $(25 \mathrm{~mm})$ horizontally, which is close to the residual radial displacement of $20 \mathrm{~mm}$ indicated by the $\mathrm{A} 2$ integrated record. However, the integrated residual radial displacement from $A 3$ was only about $13 \mathrm{~mm}$.

The integrated records from accelerometers A5 and A6 in B4-300 indicate that the peak displacement was less than $1 \mathrm{~mm}$ in the horizontal direction and less than $2 \mathrm{~mm}$ in the vertical direction. It does not seem likely that such small amplitudes would have an effect on the bunker occupants.

Additional mechanical accelerometers were added to the Febetron house for POPOVER IV; they were placed next to A2 and A3. Mechanical accelerometers A2M1 (150 g) and A2M2 $(15 \mathrm{~g})$ were placed next to $\mathrm{A} 2$, and $\mathrm{A} 3 \mathrm{M} 1(50 \mathrm{~g})$ and $\mathrm{A} 3 \mathrm{M} 2(5 \mathrm{~g})$ were placed near $\mathrm{A} 3$. In addition, A7M $(10 \mathrm{~g})$ was placed on the side of the camera at the center optical port in B4480. The mechanical accelerometers did not seem to function properly and produced questionable results. Two piezoelectric high-g pencil accelerometers (A9 and A10) were placed in the Febetron house as well, but they had electrical problems during POPOVER IV. A velocity transducer was placed for POPOVER V.

\section{POPOVER V}

The horizontal accelerations inside B4-480 ranged from 2.3 to $7.3 \mathrm{~g}$, and the vertical accelerations ranged from 3.8 to $11 \mathrm{~g}$. Inside B4-300 the horizontal accelerations ranged from 0.3 to $0.67 \mathrm{~g}$, and the vertical accelerations ranged from 0.6 to $0.85 \mathrm{~g}$. The accelerations in the Febetron house in the horizontal directions ranged from 140 to greater than $300 \mathrm{~g}$ and in the vertical direction from 210 to greater than $300 \mathrm{~g}$. Again, the accelerations were at very high frequency. The velocity gauge recorded a velocity of 2.6 $\mathrm{m} / \mathrm{s}$. No data were available for the mechanical or high-g pencil accelerometers.

The data from the accelerometers were integrated to obtain velocity records. In the case of the Febetron house, the range of the accelerometers was exceeded and some data were lost. As a result, the velocity records did not return to zero. Therefore, the velocity records were shifted so that the late-time velocities returned to the baseline. The resulting peak velocities for the Febetron house were $1.4 \mathrm{~m} / \mathrm{s}$ vertical and $0.75 \mathrm{~m} / \mathrm{s}$ horizontal. For B4-300 the peak integrated velocity was less than $0.1 \mathrm{~m} / \mathrm{s}$ in any direction.

Reference 9 was consulted to determine safe levels of velocity and acceleration for personnel occupancy. Reference 9 indicates that two types of shock exposure should be considered: impacts causing body deceleration and body vibration caused by motion of the 
structure. It states that an impact velocity of $10 \mathrm{ft} / \mathrm{s}(3 \mathrm{~m} / \mathrm{s})$ is considered to be generally safe for personnel. This type of impact might result from persons standing next to a wall, the wall moving relative to the person. Since the peak velocity in the occupied area (B4-300) was less than $0.1 \mathrm{~m} / \mathrm{s}$, this type of exposure was not a concern. The next possible exposure was the effect of horizontal motion on the stability of personnel. Reference 9 indicates that the tolerable horizontal acceleration of $0.5 \mathrm{~g}$ should be safe for nonrestrained personnel. This value is for ground shocks resulting from nuclear detonations, which are stated to be of longer duration than those from a HE explosion. As stated above, one component of the horizontal accelerations in B4-300 was $0.67 \mathrm{~g}$, which exceeds the $0.5-\mathrm{g}$ guidance. However, since the frequency of the motion was approximately $17 \mathrm{~Hz}$ and the displacements were on the order of $1 \mathrm{~mm}$, personnel safety probably will not be compromised. The vibration data in Refs. 9 and 10 indicate that the vibrational levels considered to be acceptable for restrained personnel are frequency dependent. Reference 9 lists acceptable limits of $2 \mathrm{~g}$ for frequencies less than $10 \mathrm{~Hz}$ and 5 $\mathrm{g}$ for frequencies of 10 to $20 \mathrm{~Hz}$. Therefore, the $0.67 \mathrm{~g}$ at $17 \mathrm{~Hz}$ is an acceptable level of body vibration for restrained personnel.

As far as equipment was concerned, Ref. 9 indicates that most commercially available mechanical and electrical equipment is able to sustain at least $3 \mathrm{~g}$ acceleration, while fragile equipment, such as electronic components, can sustain about $1.5 \mathrm{~g}$. This indicates that the equipment in B4-300 will probably survive. However, the equipment in B4-480 will be exposed to much higher accelerations - in this test $7.3 \mathrm{~g}$ horizontal and $11 \mathrm{~g}$ vertical. Thus, no such generic assurance can be given for equipment in B4-480. Earthquake experience data indicate that relay chatter is likely at the acceleration level in both bunkers. If relays are to be used, special consideration should be given to this effect.

\section{Summary and Conclusions}

The accelerations generated during the POPOVER series do not appear to be a threat to personnel, based on interpretation of published data and application of judgment. The only possible threat is to equipment. First, the Febetron is subjected to the highest accelerations; however, the frequency content is very high. A slight inelastic response or sliding would mitigate the effects of this high acceleration, as was shown by the aerosol can on the floor of the Febetron housing. However, the initial impulse could prove damaging to a brittle piece of equipment rigidly mounted to the floor. Feedback from the BEEF team is that the Febetron is rugged and the glass tube is encased in oil. Nevertheless, it is highly recommended that the Febetron be mounted on a engineered flexible mount to help isolate it from the high-frequency impulse. This is also true for the high-speed cameras mounted in B4-480. One concern is that air bearings (if they are used) might bottom out under the acceleration input. Care must be taken in shock-mounting equipment to be sure that the transmissibility of the isolation system does not amplify motions at critical frequencies of the equipment. In addition, a flexible mounting will, in general, increase deflections. Therefore, consideration must be given to alignment tolerances and possible interaction between structures. 


\subsection{Noise}

Impulsive noise measurements were taken at various locations in BEEF during the POPOVER test series. These measurements were used to evaluate potential personnel exposures to impulsive noise caused by the HE detonation. OSHA has set an exposure limit to impulsive or impact noise of a peak $C$-weighted level of $140 \mathrm{~dB}$, regardless of the impulse frequency or pulse duration. 6 Areas with noise above the allowable occupational exposure limits must be posted and appropriate control measures instituted (e.g., engineering controls, protective equipment, and a hearing conservation program).

Table 6-1 summarizes the sound-level measurements taken by EG\&G and LLNL Industrial Hygiene personnel in the POPOVER test series.

Table 6-1. Summary of sound level measurements (dB)

\begin{tabular}{|c|c|c|c|c|c|}
\hline Transducer location & $\begin{array}{l}\text { OPOVER I } \\
\text { (512 lb TNT) }\end{array}$ & $\begin{array}{r}\text { POPOVER II } \\
(1,000 \mathrm{lb} \text { TNT }) \\
\end{array}$ & $\begin{array}{c}\text { POPOVER III } \\
(1,000 \mathrm{lb} \text { TNT })\end{array}$ & $\begin{array}{l}\text { POPOVER IV } \\
(5,022 \mathrm{lb} \text { TNT })\end{array}$ & $\begin{array}{l}\text { POPOVER V } \\
(7,790 \text { lb TNT) }\end{array}$ \\
\hline \multicolumn{6}{|l|}{ Inside B4-300 } \\
\hline laser room (N1-A) & 109 & 84 & 113 & 128 & 130 \\
\hline laser room (N1-B) & - & - & 128 & - & 130 \\
\hline laser room (N1-C*) & - & - & - & 127 & 132 \\
\hline control room (N5-A) & 114 & 90 & 120 & 123 & 123 \\
\hline control room (N5-B) & - & - & - & - & 123 \\
\hline control room (N12*) & - & - & 113 & 121 & 130 \\
\hline utility room (N10-A) & - & - & 129 & - & 129 \\
\hline utility room (N10-B) & - & - & - & - & 126 \\
\hline utility room (N11-A*) & 125 & $>130$ & - & $>130$ & $>130$ \\
\hline utility room (N11-B*) & - & - & - & - & 134 \\
\hline entrance door (2-A) & 108 & 100 & 129 & 141 & 141 \\
\hline entrance door (2-B) & - & - & 141 & 141 & 141 \\
\hline entrance door (N8-A*) & 128 & 138 & 140 & 140 & 141 \\
\hline entrance door (N8-B*) & - & - & - & 141 & - \\
\hline \multicolumn{6}{|l|}{ Outside control trailer } \\
\hline$\left(\mathrm{N}^{*}\right)$ & 125 & 134 & 149 & 136 & 135 \\
\hline$(\mathrm{N} 10-\mathrm{A})$ & 128 & 122 & - & 134 & - \\
\hline$(\mathrm{N} 10-\mathrm{B})$ & - & - & - & 135 & - \\
\hline \multicolumn{6}{|l|}{ Inside control trailer } \\
\hline$(\mathrm{N} 4-\mathrm{A})$ & 120 & - & 142 & 134 & 132 \\
\hline (N4-B) & - & - & - & - & 132 \\
\hline (N9*) & 131 & 135 & 141 & 141 & 141 \\
\hline \multicolumn{6}{|c|}{ Camera station \#7 $(9750 \mathrm{ft}[2.97 \mathrm{~km}])$} \\
\hline$\left(\mathbf{N} 7^{*}\right)$ & 127 & 135 & 131 & 139 & 141 \\
\hline
\end{tabular}

Notes:

*Data taken from recording B\&K dosimeters and sound-level meters set to record peak values.

Dash ( $(-)$ indicates no data taken.

Because of inconsistent data from previous shots, EG\&G placed redundant dosimeters side by side at many locations.

Sound meter \#N10-A was moved from outside the control trailer to inside the B4-300 utility room on POPOVER V. 
The noise measurements given in Table 6-1 for the POPOVER $V$ test indicate that the B4-300 laser room and the control room can both be occupied with no additional hearing protection or other operational controls for explosive detonations up to the maximum permitted (1,000 lb and 5,000 lb of TNT at $15 \mathrm{ft}$ and $27 \mathrm{ft}$, respectively).

Noise measurement data collected from the utility room were insufficient to be conclusive. The single test instrument used at this location showed a relatively high reading for POPOVER I (125 dB) and exceeded the instrument's limit of $130 \mathrm{~dB}$ on the succeeding tests. During the POPOVER V overtest, three additional test instruments gave noise readings of 126 to $134 \mathrm{~dB}$. More data points should be taken for explosive detonations exceeding $1,000 \mathrm{lb}$ of TNT before a determination can be made that no hearing protection (ear plugs or ear muffs) or other operational controls are required in the utility room for tests exceeding 1,000 lb.

Noise measurements taken near the inside of the entrance door indicated that this area would be a noise hazard to personnel for HE detonations exceeding $512 \mathrm{lb}$ of TNT. Appropriate control measures must be instituted for future tests.

\subsection{Strain}

Transient dynamic strain in structural members was measured at three structural areas at $B E E F$ using six channels of instrumentation. The three structural areas were the center of the B4-300 laser room ceiling, the B4-300 access door, and the center of B4-480 ceiling. At each structural measurement area, two gauges were generally used for redundancy.

Specifically, strain gauges were installed on steel surfaces at the following locations:

$\begin{array}{lll}\text { B4-300: } & \text { center of long span of ceiling - lower rebar mat } & \text { SG1 } \\ & \text { center of long span of ceiling - lower rebar mat } & \text { SG2 } \\ \text { access door - mid span on reinforcement bar } & \text { SG5 } \\ \text { access door - mid span on reinforcement bar } & \text { SG6 } \\ \text { access door - mid span closure bolt } & \text { SG7 } \\ \text { B4-480: } & \text { center of short span of ceiling - lower rebar mat } & \text { SG3 } \\ & \text { center of short span of ceiling - lower rebar mat } & \text { SG4 }\end{array}$

The purpose of the strain measurements was to evaluate key structural elements of BEEF to ensure personnel safety and equipment protection from the blast effects of detonating $\mathrm{HE}$ outside the facility. The basis for this evaluation is a comparison between the measured and the calculated safe (dynamic elastic) allowable yield strains, ${ }^{11}$ which are listed in Table 6-2.

Table 6-2. Calculated safe (dynamic elastic) allowable yield strains

\begin{tabular}{|ll|}
\hline \multicolumn{1}{|c|}{ Location } & Strain \\
\hline B4-300 ceiling & $1667 \mu \varepsilon$ \\
B4-300 door & $1448 \mu \varepsilon$ \\
B4-480 ceiling & $2174 \mu \varepsilon$ \\
\hline
\end{tabular}


The maximum allowable ceiling strains are the transient dynamic allowable amounts which, when added to the static dead-load strains for the soil-covered ceilings of B4-300 and B4 480 , result in an elastic safety factor to yield of 1.0. The dead-load strain on the B4-300 door is zero. Safety factors less than 1.0 imply yielding or permanent structural damage; safety factors low enough could lead to structural collapse.

Table 6-3 shows the maximum (peak) positive and negative transient strains for all five tests of the POPOVER series. Numbers in bold signify the absolute maximum registered by each gauge in each test, regardless of direction. Positive strain represents tension on the surface of the structural element.

Table 6-3. Maximum positive and negative strains $(\mu \varepsilon)$ recorded from POPOVER I-V

\begin{tabular}{|c|c|c|c|c|c|c|c|c|c|c|}
\hline \multirow[b]{2}{*}{ Gauge no. (location) } & \multicolumn{2}{|c|}{$\begin{array}{c}\text { POPOVER I } \\
\text { (512 lb TNT })\end{array}$} & \multicolumn{2}{|c|}{$\begin{array}{c}\text { POPOVER II } \\
(1,000 \mathrm{lb} \text { TNT })\end{array}$} & \multicolumn{2}{|c|}{$\begin{array}{c}\text { POPOVER III } \\
(1,000 \mathrm{lb} \text { TNT })\end{array}$} & \multicolumn{2}{|c|}{$\begin{array}{l}\text { POPOVER IV } \\
(5,022 \mathrm{lb} \text { TNT })\end{array}$} & \multicolumn{2}{|c|}{$\begin{array}{l}\text { POPOVER V } \\
(7,790 \mathrm{lb} \text { TNT })\end{array}$} \\
\hline & $(+)$ & $(-)$ & $(+)$ & $(-)$ & $(+)$ & $(-)$ & $(+)$ & $(-)$ & $(+)$ & $(-)$ \\
\hline SG1 (B4-300 ceiling) & 6 & -5 & 8 & -10 & 8 & -9 & 8 & -9 & 9 & -12 \\
\hline SG2 (B4-300 ceiling) & 4 & -5 & 7 & -8 & 7 & -8 & 8 & -15 & 9 & -12 \\
\hline SG3 (B4-480 ceiling) & n/a & $\mathrm{na}$ & 280 & -120 & 330 & -100 & 540 & -150 & 180 & -150 \\
\hline SG4 (B4-480 ceiling) & $\mathbf{n} / \mathbf{a}$ & $\mathrm{n} / \mathrm{a}$ & 260 & -115 & 290 & -90 & 570 & -120 & 175 & -150 \\
\hline SG5 (B4-300 door) & 14 & -15 & 21 & -39 & 23 & 46 & 110 & -155 & 210 & -185 \\
\hline SG6 (B4-300 door) & 14 & -13 & 18 & -39 & na & n/a & na & n/a & $\mathbf{n} / \mathbf{a}$ & $\mathbf{n} / \mathbf{a}$ \\
\hline SG7 (B4-300 bolt) & $\mathbf{n} / \mathbf{a}$ & $\mathrm{n} / \mathrm{a}$ & n/a & $\mathbf{n} \mathbf{a}$ & 52 & -9 & 40 & -220 & 83 & -200 \\
\hline
\end{tabular}

Note: $\mathbf{n} / \mathbf{a}$ indicates not available.

Table 6-4 lists the safety factors for dynamic yield based on the peak measured strains listed in Table 6-3. Safety factors greater than 1.0 indicate elastic response, which by definition is below the yield point for the structural elements. Numbers in bold indicate the worst case or minimum factor of safety for dynamic yield in either tension or compression.

Table 6-4. Safety factors for dynamic yield

\begin{tabular}{|c|c|c|c|c|c|c|c|c|c|c|}
\hline \multirow[b]{2}{*}{ Gauge no. (location) } & \multicolumn{2}{|c|}{$\begin{array}{c}\text { POPOVER I } \\
\text { (512 lb TNT) }\end{array}$} & \multicolumn{2}{|c|}{$\begin{array}{r}\text { POPOVER II } \\
(1,000 \mathrm{lb} \text { TNT })\end{array}$} & \multicolumn{2}{|c|}{$\begin{array}{l}\text { POPOVER III } \\
(1,000 \mathrm{lb} \text { TNT })\end{array}$} & \multicolumn{2}{|c|}{$\begin{array}{l}\text { POPOVER IV } \\
(5,022 \mathrm{lb} \text { TNT })\end{array}$} & \multicolumn{2}{|c|}{$\begin{array}{l}\text { POPOVER V } \\
\text { (7,790 lb TNT) }\end{array}$} \\
\hline & $(+)$ & $(-)$ & $(+)$ & $(-)$ & $(+)$ & $(-)$ & $(+)$ & $(-)$ & $(+)$ & $(-)$ \\
\hline SG1 (B4-300 ceiling) & 278 & 333 & 208 & 167 & 208 & 185 & 208 & 185 & 185 & 139 \\
\hline SG2 (B4-300 ceiling) & 417 & 333 & 238 & 208 & 238 & 208 & 208 & 111 & 185 & 139 \\
\hline SG3 (B4-480 ceiling) & n/a & $\mathrm{n} / \mathrm{a}$ & 8 & 18 & 7 & 22 & 4 & 14 & 12 & 14 \\
\hline SG4 (B4-480 ceiling) & $\mathrm{n} / \mathrm{a}$ & na & 8 & 19 & 7 & 24 & 4 & 18 & 12 & 14 \\
\hline SG5 (B4-300 door) & 103 & 97 & 69 & 37 & 63 & 31 & 13 & 9 & 7 & 8 \\
\hline SG6 (B4-300 door) & 103 & 111 & 80 & 37 & $\mathrm{n} / \mathrm{a}$ & $n / a$ & n/a & $\mathrm{n} / \mathrm{a}$ & $\mathrm{n} / \mathrm{a}$ & $\mathrm{n} / \mathrm{a}$ \\
\hline SG7 (B4-300 bolt) & $\mathrm{n} / \mathrm{a}$ & n/a & $\mathbf{n a}$ & $\mathrm{n} / \mathrm{a}$ & 28 & 161 & 36 & 7 & 17 & 7 \\
\hline
\end{tabular}

Note: $(+)=$ tension and $(-)=$ compression. 


\section{POPOVER I}

This test at $512 \mathrm{lb}$ of TNT was intended as a verification test for the instrumentation to set gain levels and the dynamic ranges for the recording equipment. An early-time noise problem was identified that appears to have added noise spikes to the data for approximately the first 15 to $20 \mathrm{~ms}$. As a result, the strains recorded during this period may be somewhat artificial for POPOVER I. To determine the valid portions of each signal, Table 6-5 was constructed to examine the strain arrival times from the POPOVER series of tests.

Table 6-5. Arrival times for strain data (ms)

\begin{tabular}{|c|c|c|c|c|c|}
\hline Gauge no. (location) & $\begin{array}{r}\text { POPOVER I } \\
(512 \mathrm{lb} \text { TNT }) \\
\end{array}$ & $\begin{array}{r}\text { POPOVER II } \\
(1,000 \mathrm{lb} \text { TNT }) \\
\end{array}$ & $\begin{array}{c}\text { POPOVER III } \\
(1,000 \mathrm{lb} \text { TNT })\end{array}$ & $\begin{array}{l}\text { POPOVER IV } \\
(5,022 \mathrm{lb} \text { TNT })\end{array}$ & $\begin{array}{l}\text { POPOVER V } \\
(7,790 \mathrm{lb} \text { TNT })\end{array}$ \\
\hline SG1 (B4-300 ceiling) & -25 & 25 & 25 & 25 & 25 \\
\hline SG2 (B4-300 ceiling) & -25 & 25 & 25 & 25 & 25 \\
\hline SG3 (B4-480 ceiling) & -4 & 4 & 4 & 5 & 4 \\
\hline SG4 (B4-480 ceiling) & -4 & 4 & 4 & 5 & 4 \\
\hline SG5 (B4-300 door) & 70 & 65 & 65 & 60 & 53 \\
\hline SG6 (B4-300 door) & 70 & 65 & $\mathrm{n} / \mathrm{a}$ & n/a & na \\
\hline SG7 (B4-300 bolt) & n/a & na & 65 & 60 & 65 \\
\hline
\end{tabular}

On examining the arrival times in Table 6-5 for each channel, we assumed that the noise contamination in POPOVER I did not invalidate the strain readings from SG1, SG2, SG5, and SG6 after the cessation of the $20 \mathrm{~ms}$ of noise. Peak values for SG1 and SG2 after 20 $\mathrm{ms}$ are given in Table 6-3.

However, for SG3 and SG4 the arrival-time trend indicates that the noise contamination probably masked the data, because the arrival times should have been about $4 \mathrm{~ms}$. Additionally, SG3 and SG4 recorded a 30- $\mu \varepsilon-D C$ offset, speculated to be caused by bridged soil compaction from the blast overpressure. DC offsets for the subsequent POPOVER shots II through V were not present in the data. For these reasons, SG3 and SG4 for POPOVER I are shown as "n/a" (not available) in Table 6-3.

The values for SG5 and SG6, which were on the door, were not affected by the noise because their response started at $70 \mathrm{~ms}$ - after the noise had ceased. As expected, due to the nature of the external blast pressure, the inner surface of the door went first into tension and then into compression.

At the POPOVER I post-mortem meeting it was pointed out that, for all the strain gauges, blast overpressure acting alone should have produced a tensile (positive) strain initially followed by a compressive strain, with decaying repeated alternating cycles of tension and compression. Instead, the first amount of strain was compressive, leading to an actionitem request to check the polarity and calibration of channels SG1 and SG2. The check showed that the calibration and polarity were accurate. 12 The initial negative strain was attributed to upward arching of the ceiling, caused by the direct ground-shock loading arriving at the building side wall before the air-blast load arrived. The stiff wall-to-ceiling 
corner transmitted the bending moment to the ceiling, causing a slight upward arch and hence the initial compressive strain in the ceiling lower reinforcing bar.

\section{POPOVER II}

For this test of $1,000 \mathrm{lb}$ of TNT, the data presented no surprises. The lowest safety factor was 8 - for the camera room ceiling (SG3 and SG4). The noise effects that had occurred during POPOVER I, which were attributed to a grounding problem, were corrected before POPOVER II. At the POPOVER II post-mortem meeting, the Review Panel recommended adding a strain gauge to the door closure bolt to indicate door rebound better than SG5 or SG6 did.

In response to initially published allowable strains not being corrected for dead loads, the POPOVER Review Panel identified an action item to discover the magnitude of dynamic strain that could be safely applied to the structures. This was resolved and reported to the Review Panel chairman (see Table 6-2). ${ }^{13}$

\section{POPOVER III}

For the second test of $1,000 \mathrm{lb}$ of TNT, the measured strains were found to be well within the 20\% repeatability of the first 1,000-lb test (POPOVER II). Due to the limited number of stain-recording channels available, SG6 was disconnected in favor of a new strain gauge, SG7, which was installed on the B4-300 door closure bolt.

\section{POPOVER IV}

As expected, for this single $5,022 \mathrm{lb}$ of TNT shot, the strain levels were higher than in POPOVER III. The lowest dynamic-yield safety factor was 4 - for the roof of the camera room (SG3 and SG4).

\section{POPOVER $V$}

In this shot, multiple distinct charges of $1,256 \mathrm{lb}$ and $6,534 \mathrm{lb}$ of TNT were detonated simultaneously. The strain levels were not always greater than for the single 5,022-lb shot (POPOVER IV). This anomaly is considered to be a result of shot interference that occurs when the points of interest are close to multiple charges separated by some distance. For example, the strain gauges in the camera room roof (close to the detonation points), SG3 and SG4, produced positive strains that were roughly one-third that of the previous test. The negative strains were of the same amplitude but longer duration. Farther away, at B4-300, strain gauges SG1, SG2, SG5, and SG7 were less affected by the shot interference because the multiple charges behaved more like a single charge. Their levels were only slightly greater than those for POPOVER IV. 


\section{Summary and Conclusions}

In general, as the loading on a structure increases, the danger of collapse will not occur until significantly after structural damage (yielding) occurs. Early signs of yielding can be identified by measuring the total (dead load plus transient) strain in key structural elements, such as the bunker ceilings in the case of BEEF, and comparing the total measured strain with established yield strain levels. For BEEF the dead-load ceiling strains could not be measured because strain gauges would have had to have been put on the rebar when the facilities were constructed, which was more than 40 years ago.

However, new strain gauges were applied to the BEEF structures just before the POPOVER series. Because they were installed after the dead loads were applied, the new strain gauges measured only the transient dynamic strains and the changes in the static dead-load strains during each certification shot. From the weight of the soil cover and the ceiling, the dead-load strains were calculated. Maximum allowable transient-strain levels were then obtained by subtracting the calculated dead-load strains from the calculated equivalent static-yield strain levels established for each structure.

Examination of the transient-strain safety factors to yield in Table 6-4 shows no evidence of structural damage to Bunkers B4-480 or B4-300 or failure of the door of B4-300 for the HE levels and shot locations used to qualify BEEF during the POPOVER tests. Structural damage would be indicated by safety factors less than 1.0. Personnel and equipment are protected by the structural integrity of B4-300. The POPOVER tests show that transient strains 111 times greater for the ceiling and 7 times greater for the door would be required to cause structural damage (yielding). Likewise, equipment such as cameras in B4-480 will be protected by the building structural integrity. Transient strains 4 times higher than measured would be required to cause structural damage in B4-480.

No significant permanent strain offsets were observed in the strain records during each POPOVER test. Normal "temperature" cracks in the concrete interior structure of B4-300 were observed before POPOVER. The ends of these cracks were taped before POPOVER I so that any further cracking due to the POPOVER tests could be observed. No cracks propagated beyond the tape or broke the tape as a result of the POPOVER series. From this we can conclude that the pre-POPOVER cracking was due to normal aging and settling of concrete. For the HE weights and locations tested in the POPOVER series, the structural response of BEEF is elastic, which precludes permanent structural damage.

\subsection{Blast Thermal Effects and Fragment Protection}

Although not actually evaluated, the construction features of BEEF are considered adequate to protect personnel located in B4-300 from all blast thermal effects and any primary fragments (excluding shaped-charge jet effects) resulting from the detonation of thousands of pounds of conventional $\mathrm{HE}$ charges. These construction features include an earth berm $15 \mathrm{ft}(4.5 \mathrm{~m})$ between the experimental test site and the personnel-occupied B4-300, which has $10 \mathrm{ft}(3 \mathrm{~m})$ of compacted earth fill on top of the bunker roof. The reinforced-concrete bunker ceiling is $30 \mathrm{in}$. $(0.75 \mathrm{~m})$ thick, and the walls are $24 \mathrm{in} .(0.5 \mathrm{~m})$ thick. 
Fragment-penetration analysis was not specifically performed during the POPOVER test series. However, published data in Fig. 8-6 of Ref. 7 indicate that the maximum penetration of a 100-oz. (3.1 kg) primary fragment with a striking velocity of $2,000 \mathrm{ft} / \mathrm{s}$ $(610 \mathrm{~m} / \mathrm{s})$ - a reasonable worst-possible case - would be approximately 64 in. $(1.6 \mathrm{~m})$ in sand. Smaller, higher velocity fragments are more easily stopped. Although the fragmentpenetration properties (grain size, compaction, and mass) of the sand tested and the earth fill used at BEEF may differ slightly, the Review Panel believes that these differences are insignificant and that the fragment-penetration properties for sand and the compacted earth fill at BEEF are generally comparable. The protection provided by the earth berm, the total compacted-earth-fill thickness of 120 in. $(3 \mathrm{~m})$, and the reinforced concrete bunker provide substantial additional fragment protection to personnel.

Experiments involving shaped charges, because of their unique penetrating-jet characteristics, must be carefully reviewed individually and adequately controlled. Sufficient shielding is needed to prevent the shaped charge from penetrating the facility or to control its direction away from the underground bunkers and support equipment. A miscalculation of the penetration capability of a shaped-charge experiment can cause a manned bunker to be penetrated. ${ }^{14}$

Blast effects and fragment protection for auxiliary experimental equipment were not reviewed in any great depth because specific future equipment requirements could not be predicted. However, in POPOVER V an empty nitrogen tube trailer was placed below grade and protected from low-angle high-velocity fragments; it was damaged as described in Section 6.1. The remote possibility exists that one or more high-angle terminal-velocity fragments might penetrate a tube in a tube trailer, which could release stored nitrogen. It is recommended that each piece of unprotected equipment (such as a nitrogen tube trailer) that will be present during future explosive experiments be reviewed for shrapnel hazards and consequences. 


\section{Section 7}

\section{Findings and Recommendations}

The POPOVER test series produced evidence to show that BEEF exceeds the DOE requirements for explosives facilities and is safe for the presence of personnel in the control bunker (Bunker B4-300) during future experiments and that the facility will function as intended. In general, the Review Panel recommends a case-by-case evaluation of future experiments for issues or features not considered during the POPOVER series. Specific findings and recommendations of the Review Panel are grouped into (1) personnel safety issues and (2) facility issues.

\subsection{Personnel Safety}

The following are the Review Panel's findings and recommendations regarding the safety of personnel at BEEF in Bunker B4-300. It is the Review Panel's understanding that personnel will not be allowed in B4-480 during explosives testing.

\begin{tabular}{|ll|}
\hline Potential hazard & Finding and recommendation \\
\hline Blast pressure & $\begin{array}{l}\text { Maximum pressures measured in Bunker B4-300 and the } \\
\text { control trailer for the POPOVER series were below the } \\
\text { personnel limit of 0.055 psig (380 Pa) established in } \\
\text { Ref. } 4 \text { for nonessential personnel. } \\
\text { Recommendations: } \\
\text { Generate an HE placement chart to ensure that the effects } \\
\text { of future experiments fall within the POPOVER } \\
\text { experience. }\end{array}$ \\
$\begin{array}{l}\text { Establish and implement administrative procedures to } \\
\text { eliminate the possibility of the pressure increasing in }\end{array}$ \\
Bunkers B4-300 and B4-480 during future experiments. \\
Ground acceleration & $\begin{array}{l}\text { Accelerations measured in Bunker B4-300 for all the tests } \\
\text { in the POPOVER series are not a threat to personnel based } \\
\text { on interpretation of the limits established in Refs. } 9 \text { and } \\
\text { 10. }\end{array}$
\end{tabular}




\begin{tabular}{|c|c|}
\hline Potential hazard & Finding and recommendation \\
\hline \multirow[t]{4}{*}{ Impulsive noise } & $\begin{array}{l}\text { In general, BEEF met OSHA noise requirements. Noise } \\
\text { intensity near the Bunker B4-300 door was greater than } \\
140 \mathrm{~dB} \text {. }\end{array}$ \\
\hline & Recommendations: \\
\hline & $\begin{array}{l}\text { Establish and implement positive measures to prevent } \\
\text { injury to personnel due to noise. }\end{array}$ \\
\hline & $\begin{array}{l}\text { For charge sizes larger than those in the POPOVER } \\
\text { series, evaluate the effects of charge size on noise } \\
\text { intensity in Bunker B4-300. }\end{array}$ \\
\hline Structural collapse & $\begin{array}{l}\text { Measured strains in the ceiling of Bunker B4-300 for the } \\
\text { POPOVER series were } 111 \text { times less than that required } \\
\text { for permanent deformation. This precludes structural } \\
\text { collapse. }\end{array}$ \\
\hline \multirow[t]{6}{*}{ Confined space } & $\begin{array}{l}\text { Bunker B4-300 has only one personnel door. Before } \\
\text { detonating explosives, the air supply is closed off } \\
\text { manually with valves located outside the bunker. }\end{array}$ \\
\hline & Recommendations: \\
\hline & $\begin{array}{l}\text { Install oxygen-deficiency sensors in Bunkers B4-300 and } \\
\text { B4-480. }\end{array}$ \\
\hline & $\begin{array}{l}\text { Establish operational limits on length of time personnel } \\
\text { are allowed to stay in the closed bunker. }\end{array}$ \\
\hline & $\begin{array}{l}\text { Prepare an emergency response plan to address } \\
\text { unforeseen safety threats that might cause asphyxiation of } \\
\text { personnel (for example, rupture of the tube trailer). }\end{array}$ \\
\hline & $\begin{array}{l}\text { Relocate the air supply and return valves inside the } \\
\text { bunker. }\end{array}$ \\
\hline $\begin{array}{l}\text { Blast thermal effects } \\
\text { and fragment } \\
\text { protection }\end{array}$ & $\begin{array}{l}\text { The construction features of Bunker B4-300 are adequate } \\
\text { to protect personnel from thermal flux effects and any } \\
\text { fragments resulting from a detonation of thousands of } \\
\text { pounds of } \mathrm{HE} \text { charges. }\end{array}$ \\
\hline
\end{tabular}

\subsection{Facility and Equipment}

For the POPOVER series, acceleration, strain, and pressure predictions were generally conservative - i.e., measured data were lower than the estimates. However, for future tests the Review Panel makes the following recommendations:

- Determine estimated pressure intensities and durations and compare them with those measured in the POPOVER series. 
- Evaluate the effects on the facility of shrapnel from shaped charges and provide sufficient shielding for the underground bunkers and support equipment.

- Evaluate the effects of high accelerations on any diagnostic equipment (e.g., Febetrons and streak cameras) that will be used in future shaped-charge experiments that was not in place for the POPOVER series.

- Any modification of the site or bunker configuration that might adversely affect the safe operation of the facility should not be permitted without recertification and/or design review.

- Evaluate each piece of unprotected research equipment during future experiments to determine worst-case scenarios and consequences in the event they could be damaged by blast or fragments. 


\section{Section 8}

\section{References}

1. K.Winer, L. Haselman, L. Simmons, J. Page, J. Wobser, K. Pedersen, ShapedCharge Scaling Project (Proposal to Extend Site 300's Diagnostic Capabilities to Experiments Involving Very Large Explosive Charges), Lawrence Livermore National Laboratory, Livermore, CA, internal report (March 29, 1995).

2. U.S. Department of Energy, General Design Criteria, U.S. Department of Energy, Germantown, MD, Order 6430.1A (1989).

3. U.S. Department of Energy, Explosives Safety Manual, U.S. Department of Energy, Germantown, MD, M 440.1-1 (1995).

4. U.S. Department of Defense, DoD Ammunition and Explosive Safety Standards, U.S. Department of Defense, Arlington, VA, 6055.9-STD (1992), Chapter 4, Section C. 2 and Chapter 5, Section 4.a.

5. U.S. Department of the Air Force, Explosives Safety Standards, U.S. Air Force, Washington, DC, AF Regulation 127-100 (1990).

6. Occupational Noise Exposure, U.S. Code of Federal Regulations, 29 CFR 1910.95 (1994).

7. EG\&G Energy Measurements, POPOVER Certification Test Series Final Engineering Report, EG\&G Energy Measurements, Las Vegas, NV, SM:95E-179-38 (1995) [3 books].

8. R. Heinle and T. Stubbs, "POPOVER Data Report," Lawrence Livermore National Laboratory, Livermore, CA, UCRL-ID-123701 (1996).

9. U.S. Department of Defense, Structures to Resist the Effects of Accidental Explosion, U.S. Department of Defense, Arlington, VA, TM 5-1300/NAVFAC P-397/AFR88-22 (1990).

10. W.W. Banks, A Review of Unclassified Data Relating to Survivability of Manned Bunkers Under Explosive Attack, Lawrence Livermore National Laboratory, Livermore, CA, UCID-20241 (1984).

11. C.Y. "John" King, BEEF Big Explosives Experimental Facility Certification Issues, Lawrence Livermore National Laboratory, Livermore, CA, internal report (March 1995).

12. R.A. Robinson to B.W. Bellow, "POPOVER Strain Gage SG-12 Shunt Resistance Verification," EG\&G Energy Measurements, Las Vegas, NV, memorandum SM:95E179-27 (May 31, 1995). 
13. J. Pastrnak to T. Davito, "Review of Maximum Allowable Strains in B4-300 and B4-480 Roofs," Lawrence Livermore National Laboratory, Livermore, CA, memorandum (June 2, 1995).

14. Lawrence Livermore National Laboratory Hazards Control Department, Bunker 851 Incident Report, Lawrence Livermore National Laboratory, Livermore, CA, internal report (December 31, 1968). 


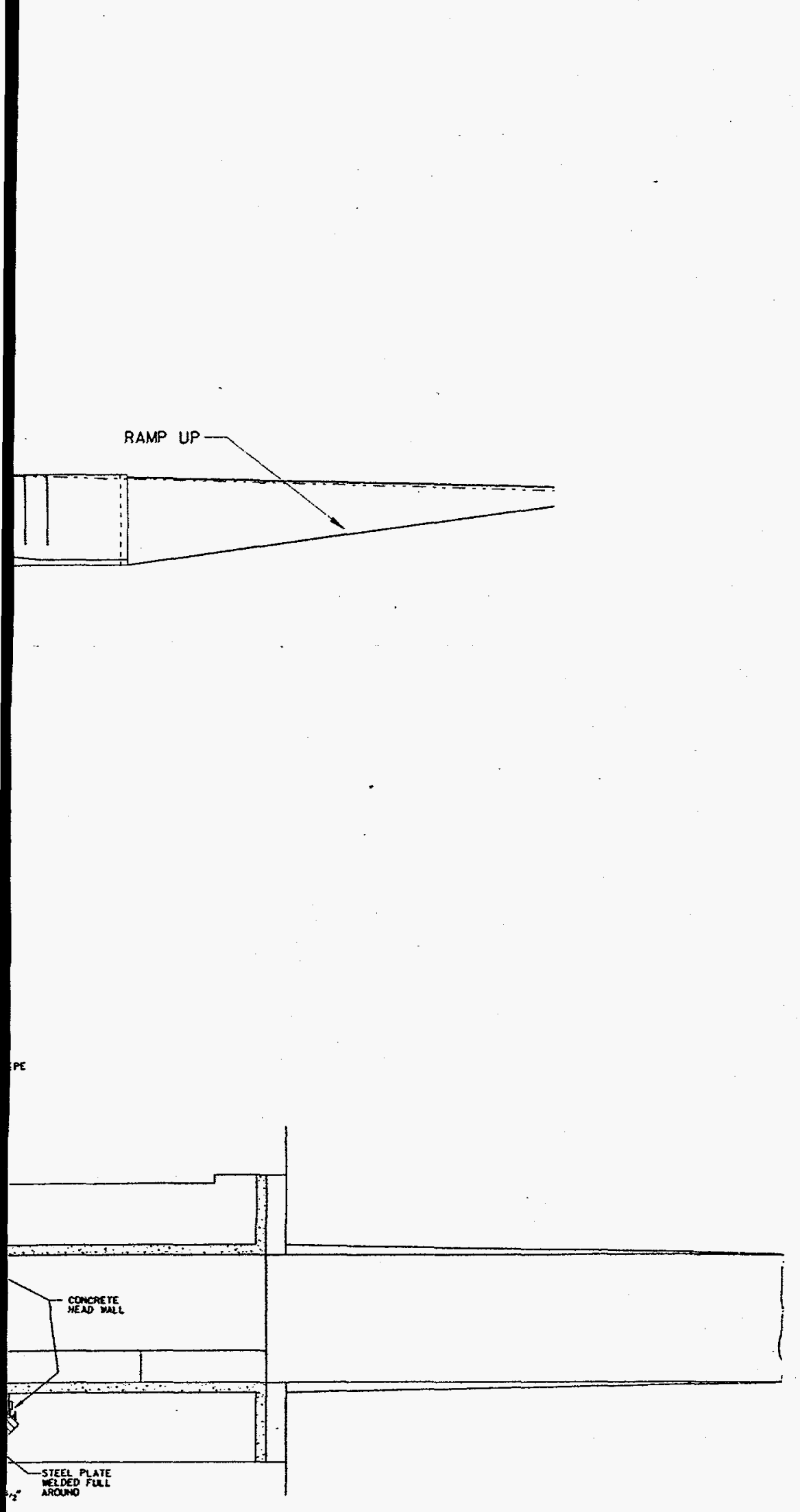

